\title{
General mirror pairs for gauged linear sigma models
}

\author{
Paul S. Aspinwall and M. Ronen Plesser \\ Departments of Mathematics and Physics, Duke University, \\ Box 90320, Durham, NC 27708-0320, U.S.A. \\ E-mail: psa@cgtp.duke.edu, plesser@cgtp.duke.edu
}

\begin{abstract}
We carefully analyze the conditions for an abelian gauged linear $\sigma$-model to exhibit nontrivial IR behavior described by a nonsingular superconformal field theory determining a superstring vacuum. This is done without reference to a geometric phase, by associating singular behavior to a noncompact space of (semi-)classical vacua. We find that models determined by reflexive combinatorial data are nonsingular for generic values of their parameters. This condition has the pleasant feature that the mirror of a nonsingular gauged linear $\sigma$-model is another such model, but it is clearly too strong and we provide an example of a non-reflexive mirror pair. We discuss a weaker condition inspired by considering extremal transitions, which is also mirror symmetric and which we conjecture to be sufficient. We apply these ideas to extremal transitions and to understanding the way in which both Berglund-Hübsch mirror symmetry and the Vafa-Witten mirror orbifold with discrete torsion can be seen as special cases of the general combinatorial duality of gauged linear $\sigma$-models. In the former case we encounter an example showing that our weaker condition is still not necessary.
\end{abstract}

Keywords: Conformal Field Models in String Theory, Superstring Vacua

ARXiv EPrint: 1507.00301 


\section{Contents}

1 Introduction $\quad 1$

2 The gauged linear $\sigma$-model $\quad 3$

2.1 The pointsets $\mathscr{A}$ and $\mathscr{B} \quad 3$

2.2 The hyperplane conditions 5

2.3 The low-energy limit 6

2.4 Geometry 8

2.5 The GKZ determinant for $\mathscr{B} \quad 9$

2.5.1 The generic $\mathbb{C}^{*}$ orbit 9

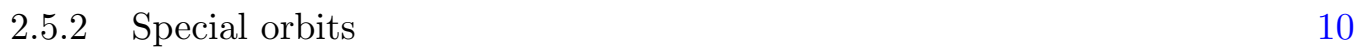

$\begin{array}{ll}2.5 .3 \text { A sufficient condition for compactness } & 12\end{array}$

$\begin{array}{lll}2.6 & \text { The determinant for } \mathscr{A} & 13\end{array}$

$\begin{array}{lll}2.6 .1 & \text { The primary component } & 13\end{array}$

$\begin{array}{lll}2.6 .2 & \text { Other components } & 15\end{array}$

$\begin{array}{lll}2.7 & \text { Mirror symmetry } & 17\end{array}$

$\begin{array}{lll}2.8 & \text { Extremal transitions and breaking the reflexive condition } & 18\end{array}$

3 Examples $\quad 20$

3.1 A non-reflexive example 20

3.2 Extremal transitions 21

$\begin{array}{lll}3.2 .1 & \mathbb{P}^{5} \mid 42 & 21\end{array}$

$\begin{array}{lll}3.2 .2 & \mathbb{P}_{\{2,1,1,1,1,1\}}^{5} \mid 43 & 23\end{array}$

$\begin{array}{ll}3.2 .3 \mathbb{P}^{6} \mid 322 & 24\end{array}$

4 Ignoring interior points $\quad 24$

\begin{tabular}{ll}
4.1 & Berglund-Hübsch mirror symmetry \\
\hline
\end{tabular}

$\begin{array}{lll}4.1 .1 & \text { A reflexive model } & 25\end{array}$

4.1.2 A non-reflexive version 26

$\begin{array}{lll}4.2 & \text { Vafa-Witten mirror symmetry } & 27\end{array}$

\section{Introduction}

Witten's abelian gauged linear $\sigma$-model [1] has been a powerful tool in mirror symmetry for many years $[2,3]$. For a Calabi-Yau space $X$ determined as a complete intersection in a toric variety, the gauged linear $\sigma$-model provides a UV safe model whose IR behavior is governed by the superconformal field theory determined by the non-linear $\sigma$-model with target space $X$. The combinatorial duality of Batyrev and Borisov [4, 5] relating mirror 
families can be stated $[6,7]$ as a duality relating gauged linear $\sigma$-models with identical IR physics, including an explicit mapping of the parameters.

Conventionally the focus has been on the geometry of mirror symmetry. The desired Calabi-Yau $X$ is a subvariety of a toric variety, which is assumed to be Gorenstein. However, both from a physics and a mathematical point of view, it is necessary to widen one's perspective from the geometry of varieties, as has long been known [8].

From a physics perspective, mirror symmetry relates isomorphic $N=(2,2)$ superconformal field theories with some fixed central charge $3 d$ and integral $\mathrm{U}(1)_{R}$ charges. Such an object may, or may not, arise as a nonlinear $\sigma$-model on a Calabi-Yau $d$-fold. In either case, it is equally useful for compactifying a string model. Similarly, the mirror of a Calabi-Yau $d$-fold may, or may not, be another Calabi-Yau $d$-fold. In the context of gauged linear $\sigma$-models a given model may, or may not, exhibit a geometric phase corresponding to a large-radius Calabi-Yau $d$-fold. In general, it is probable (although currently no examples are known) that a gauged linear $\sigma$-model can have no geometric description anywhere in its parameter space. ${ }^{1}$

From the mathematical perspective, mirror symmetry is an equivalence between two triangulated categories (or dg-categories). The essentially structure of a Calabi-Yau $d$ fold is thus its underlying Calabi-Yau category structure. Now, an abstract Calabi-Yau category of dimension $d$ may, or may not, be expressible as the derived category of some actual Calabi-Yau $d$-fold. This is really the exact same statement as the above paragraph. Thus, in this paper we take the perspective that one is primarily interested in generating $N=(2,2)$ superconformal field theories with spectral flow, or equivalently, Calabi-Yau categories. Whether or not such a model exhibits a geometric limit in any way is a difficult question to answer and will be of secondary importance to us.

The main focus of this paper is a precise condition for the nonsingularity of a gauged linear $\sigma$-model without reference to a geometric phase. We try to be rigorous and complete. The data provided is a set of chiral fields, an abelian gauge group (which may have discrete factors) and a superpotential. Out of these data come two pointsets $\mathscr{A}$ and $\mathscr{B}$ that live in dual lattices. We demand that the theory defined by these pointsets be nonsingular for generic values of the parameters. Not surprisingly, in theorem 7 , we recover reflexivity of the pointsets as a sufficient condition. However, it is clear that reflexivity is not necessary.

Geometrically an extremal transition (or generalized conifold transition) connects two topologically distinct models by a blow-down followed by a deformation. It has long been realized that this is easy to implement in terms of the gauged linear $\sigma$-model data [9] by removing points from $\mathscr{A}$ to shrink its convex hull and then adding points to $\mathscr{B}$ to increase its convex hull. The same mechanism works whether there is a geometrical interpretation or not and we will still refer to it as an extremal transition. In the four-dimensional spacetime theory (for $d=3$ ) these are Coulomb-Higgs transitions that can be analyzed in quantum field theory in a limit in which gravity decouples. In this paper we will use such transitions to motivate a less restrictive condition for nonsingularity of the IR physics and the associated extension of the mirror duality given by conjecture 10 .

\footnotetext{
${ }^{1}$ We know models that do not have a geometric phase but in all known cases there is a mirror model that does exhibit such a phase.
} 
The extremal transitions between models with geometric phases and those without are quite interesting and we give some examples. These will also connect reflexive pairs with nonreflexive pairs thus showing that one must drop the reflexive condition to fully understand the connected web of compactifications.

In section 2 we define the data that goes into constructing a gauged linear $\sigma$-model. Building on the work of [6] we see that the question of singularities is very closely tied with Gelfand, Kapranov and Zelevinski's work on discriminants [10]. In particular, for both the chiral and twisted chiral moduli spaces we have a combinatorial construction of the set of singular theories based on faces of convex hulls of pointsets. This allows us to formulate theorem 7 which states sufficient (but not necessary) conditions for a model to be nonsingular for generic moduli.

We give examples of consistent pointsets $\mathscr{A}$ and $\mathscr{B}$ in section 3 . In particular, we give a mirror pair that does not consist of a reflexive pair. Dropping points on the boundary on the convex hull of these pointsets to violate the conditions of theorem 7 leads to extremal transitions. We discuss some interesting extremal transitions from models with a geometrical description to ones without. In particular we see how non-geometrical Gepner models fit into the connected web of $N=(2,2)$ superconformal field theories.

In section 4 we consider more examples which are simplified by dropping points interior to the convex hull. This gives a simple description of Berglund and Hübsch's mirror symmetry construction [11]. We then reinterpret this example as a nonreflexive model which violates the conditions of the conjecture and yet remains nonsingular. This proves that even our weakened conditions in the conjecture are still too strong. Finally we describe Vafa and Witten's discrete torsion mirror pair [12].

\section{The gauged linear $\sigma$-model}

\subsection{The pointsets $\mathscr{A}$ and $\mathscr{B}$}

Let us consider a general setting for gauged linear $\sigma$-model and describe how the data describing the model can be written in the language of toric geometry. We base this on the construction in [6].

The data determining our family of $N=(2,2)$ two-dimensional models is:

- A set of $n$ chiral superfields $\left\{x_{1}, \ldots, x_{n}\right\}$.

- An abelian gauge group $G$ acting effectively and diagonally on $\left\{x_{1}, \ldots, x_{n}\right\}$.

- A family of worldsheet superpotentials $W$, which are polynomials in $\left\{x_{1}, \ldots, x_{n}\right\}$ invariant under $G$.

For consistency of notation with the toric construction, we will use $G$ to denote the complexified gauge group. That is, $G$ is of the form $\left(\mathbb{C}^{*}\right)^{r} \times \Gamma$ for some finite group $\Gamma$.

We can present $G$ and its action on $\left\{x_{1}, \ldots, x_{n}\right\}$ in the usual toric language as follows. First introduce the character group of $G$ :

$$
\widehat{G}=\operatorname{Hom}_{\mathbb{Z}}\left(G, \mathbb{C}^{*}\right),
$$


which is isomorphic to $\mathbb{Z}^{r} \oplus \Gamma$. Note that if $\mathfrak{g}$ is the Lie algebra of $G$ then

$$
\widehat{G} \otimes \mathbb{C}=\mathfrak{g}^{*} .
$$

The diagonal $G$-action on $\left\{x_{1}, \ldots, x_{n}\right\}$ gives an obvious eigenvalue map $q: G \rightarrow\left(\mathbb{C}^{*}\right)^{n}$. If the action is effective, $q$ is injective. Applying $\operatorname{Hom}\left(-, \mathbb{C}^{*}\right)$ to $q$ gives a presentation of $\widehat{G}$ :

$$
0 \longrightarrow M \stackrel{A^{t}}{\longrightarrow} \mathbb{Z}^{n} \stackrel{Q}{\longrightarrow} \widehat{G} \longrightarrow 0
$$

where $M$ is a lattice of rank $d=n-r$ and $A$ is a $d \times n$ matrix. The map from $\mathbb{Z}^{n}$ to the free part of $\widehat{G}$ is given by the charge matrix for the $\left(\mathbb{C}^{*}\right)^{r}$-action.

Let $N$ denote the dual lattice of $M$ and let $T_{N}=N \otimes_{\mathbb{Z}} \mathbb{C}^{*}$. The matrix $A$ can be viewed as having columns giving the coordinates of $n$ points in $N$. In this way we identify the superfields $\left\{x_{1}, \ldots, x_{n}\right\}$ with $n$ points in $N$. Let $\mathscr{A}$ denote this pointset. Thus from (2.3) we now have the short exact sequence familiar from toric geometry [13]

$$
1 \longrightarrow G \stackrel{Q^{t}}{\longrightarrow}\left(\mathbb{C}^{*}\right)^{\mathscr{A}} \stackrel{A}{\longrightarrow} T_{N} \longrightarrow 1
$$

Now consider the superpotential. Suppose there are $m$ terms in this polynomial. Each monomial represents a vector in $\mathbb{Z}^{n}$ given by the list of powers in the monomial. Thus the powers of in the monomials in the superpotential define a matrix giving a map

$$
P: \mathbb{Z}^{m} \rightarrow \mathbb{Z}^{n}
$$

The superpotential is invariant under the $G$-action, which amounts to the statement $Q P=$ 0 . The map $P$ must therefore factor through the kernel of $Q$ given by (2.3). This defines a map

$$
B: \mathbb{Z}^{m} \rightarrow M,
$$

and the map in (2.5) is given by the composition $P=A^{t} B$.

The columns of the $d \times m$ matrix $B$ give a set of $m$ points in the lattice $M$. Let $\mathscr{B}$ denote this pointset. The condition that $W$ is polynomial implies

$$
\mathscr{B} \subset \operatorname{Cone}(\operatorname{Conv} \mathscr{A})^{\vee} \text {. }
$$

Each element $\beta \in \mathscr{B}$ determines a monomial, which we denote

$$
x^{\beta}=\prod_{\alpha \in \mathscr{A}} x_{\alpha}^{\langle\beta, \alpha\rangle},
$$

and our family of superpotentials is

$$
W=\sum_{\beta \in \mathscr{B}} b_{\beta} x^{\beta},
$$

where $b_{\beta} \in \mathbb{C}$. 


\subsection{The hyperplane conditions}

The combinatorial data determine a family of gauged linear $\sigma$-models. In general, these will exhibit trivial IR dynamics. We are interested in using the IR limit of such theories to construct superconformal field theories with integral $R$ charges. In this section we determine the conditions on the data under which this is the case. In models with a geometric interpretation this is equivalent to the Calabi-Yau condition. A $(2,2)$ superconformal field theory exhibits a chiral $\mathrm{U}(1)_{R}$ symmetry that is part of the superconformal algebra. Following [1] we assume that a gauged linear $\sigma$-model will determine a nontrivial superconformal field theory in the IR if it exhibits a chiral $R$-symmetry that is non-anomalous, hence preserved along the RG flow.

The eigenvalues of an $R$-symmetry which acts diagonally on the $x_{\alpha}$ 's give a map $R: \mathbb{Q} \rightarrow \mathbb{Q}^{\mathscr{A}}$. Define $\nu=A R$ to get the diagram

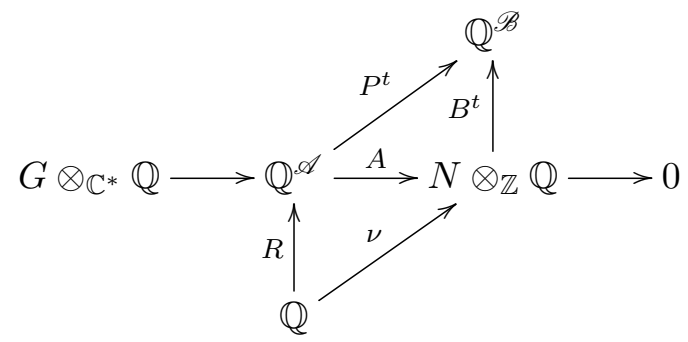

An unbroken $R$-symmetry will exist in our model if we can assign rational charges to the chiral superfields such that each term in the superpotential has charge one. That means

$$
B^{t} \nu=P^{t} R=\mathbf{1}_{m}
$$

where $\mathbf{1}_{m}$ is a column vector of $m$ ones. In other words, if we use $\nu$ to denote the image $\nu(1)$ in $N \otimes \mathbb{Q}$, we have

$$
\langle\beta, \nu\rangle=1, \quad \forall \beta \in \mathscr{B} .
$$

Our interest here is in superconformal field theories suitable for constructing string vacua with spacetime supersymmetry. This imposes an additional condition, namely that the $R$ charges of all gauge invariant chiral operators be integral. Such operators are associated by (2.8) to points in $M$. Thus $\nu \in N$.

Now we turn our attention to a similar condition for $\mathscr{A}$. The axial R-symmetry will be non-anomalous if for each generator of the continuous part $\left(\mathbb{C}^{*}\right)^{r} \subset G$ the sum of the charges of the chiral fields vanishes [1].

If the finite group $\Gamma$ is nontrivial, we again have an additional restriction from the requirement that the $R$ charges are integral. In particular, we consider the requirement that the right-moving $R$ charge of states in sectors twisted by elements of $\Gamma$ are integral. The argument closely follows a similar construction in [14]. Consider an element $g \in \Gamma$ of order $N$ acting as

$$
x_{\alpha} \mapsto e^{\frac{2 \pi i w_{\alpha}}{N}} x_{\alpha}
$$

Working in the extreme UV we can describe the vacuum in the NS sector twisted by $g$ using free fields. The right-moving $R$ charge can be written in terms of the free field currents 
$\bar{\psi}_{\alpha} \psi_{\alpha}$ and $\bar{\gamma}_{\alpha} \gamma_{\alpha}$ acting on the right- and left-moving fermions in the chiral multiplet $x_{\alpha}$

$$
R_{\text {right }}=\sum_{\alpha}\left(R_{\alpha}-1\right) \bar{\psi}_{\alpha} \psi_{\alpha}+R_{\alpha} \bar{\gamma}_{\alpha} \gamma_{\alpha}
$$

(These symmetries are of course separately broken by the interactions.) In the NS sector twisted by $g$ the vacuum carries charge $-w_{\alpha} / N(\bmod 1)$ under $\bar{\psi}_{\alpha} \psi_{\alpha}$ and charge $w_{\alpha} / N$ (mod 1) under $\bar{\gamma}_{\alpha} \gamma_{\alpha}$. Inserting this we see that the $R$ charge of the vacuum (and hence of the excitations above it by gauge-invariant fields with integral $R$ charge) is

$$
\left\langle g\left|R_{\text {right }}\right| g\right\rangle=\sum_{\alpha}\left(w_{\alpha} / N\right) \bmod 1
$$

Together with the requirement that the anomaly vanishes, $(2.15)$ is equivalent to the statement that the monomial $\prod_{\alpha \in \mathscr{A}} x_{\alpha}$ is invariant under all of $G$. (This monomial may, or may not, be in the superpotential.)

This can also be phrased as the condition $Q \mathbf{1}_{n}=0$. We therefore have a diagram inducing a map $\mu$ :

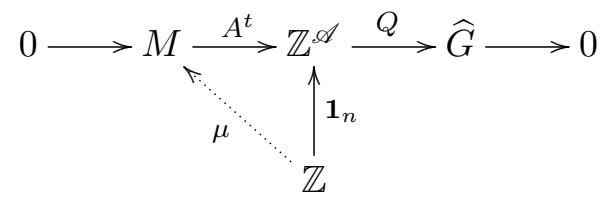

Again, let $\mu$ denote the image of $\mu(1)$ in $M$. The above diagram then implies

$$
\langle\mu, \alpha\rangle=1, \quad \forall \alpha \in \mathscr{A}
$$

The gauged linear $\sigma$-models of interest are thus those for which $\mathscr{A}$ and $\mathscr{B}$ both lie in "primitive" hyperplanes in the sense that we have lattice vectors $\mu$ and $\nu$ defining the hyperplanes $(2.12)$ and $(2.17) .^{2}$

\subsection{The low-energy limit}

A collection of data satisfying this condition will determine a family of gauged linear $\sigma$ models, whose extreme IR limits will be a family of $\mathscr{N}=(2,2)$ superconformal field theories of central charge given by [8]

$$
\frac{1}{3} c=d-2\langle\mu, \nu\rangle
$$

(This is also the dimension of the associated Calabi-Yau category.) The deformation theory is simple and well understood. The deformation space splits locally as a product of two complex special Kähler spaces associated to deforming the action by the top components of chiral (resp. twisted chiral) multiplets of charge $(1,1)$ (resp. $(1,-1))$ under the left- and right-moving $R$-symmetry. Deformations are not obstructed: all supersymmetric marginal deformations are truly marginal.

\footnotetext{
${ }^{2}$ This condition also shows that the points $\alpha \in \mathscr{A}$ are primitive generators of the rays in the toric fan. This means we avoid having to use any stacky construction of the form described in [15].
} 
With our conventions, the deformations associated to chiral multiplets will be holomorphically parametrized by a choice of the coefficients $b_{\beta} \in \mathbb{C}^{m}$ determining the superpotential. Since deformations of the kinetic terms are irrelevant in the IR theory, changes in these that can be undone by field redefinitions consistent with the R-symmetry are redundant. We will here restrict attention to diagonal field redefinitions contained in $\left(\mathbb{C}^{*}\right)^{n}$. These act on $\mathbb{C}^{m}$ through an action determined by $P$, whose rank shows that the space of orbits has dimension $m-d$. As is standard, we define a compact model for this space of deformations as the toric variety associated to the secondary fan of $\mathscr{B}$. We denote this compact space $\mathscr{M}_{B}$.

Deformations associated to twisted chiral multiplets will be parametrized by the FayetIliopoulos coefficients and $\theta$ angles for the gauge group, both of which live in $\mathfrak{g}_{\mathbb{R}}^{*}$. The combination $\tau=i \rho+\frac{\theta}{2 \pi} \in \mathfrak{g}^{*}$ will give local holomorphic coordinates. This space $\mathscr{M}_{A}$ will thus have dimension $r=\operatorname{rank} G=n-d$. Again we define $\mathscr{M}_{A}$ to be the toric variety associated to the secondary fan of $\mathscr{A}$. Locally $q=\exp (2 \pi i \tau)$ are good coordinates.

Note we make no claim that $\mathscr{M}_{B}$ is the moduli space of chiral deformations, nor $\mathscr{M}_{A}$ the moduli space of twisted chiral deformations. The dimensions will typically be incorrect. For $\mathscr{M}_{B}$ this may be because of non-polynomial deformations, non-toric field redefinitions, or simply because we choose to not include a point in $\mathscr{B}$ that could have been used as a monomial in $W$. Similar statements apply to $\mathscr{M}_{A}$ and $\mathscr{A}$.

For special values of the parameters of either type the low-energy theory will be singular. Because of the factorization properties the singular locus will locally be the product of two complex subvarieties of $\mathscr{M}_{B}$ and $\mathscr{M}_{A}$ (although at intersections of these the singularity type changes in interesting ways this will not concern us here). We will partially characterize these subvarieties below. In particular, we wish to formulate conditions on the combinatorial data determining the family sufficient to ensure that the generic member is not singular.

We can predict the singular locus by considering the space of gauge-inequivalent supersymmetric field configurations. These are classically determined by constant expectation values for the scalars $x_{\alpha}$ in the chiral multiplets and a field $\sigma \in \mathfrak{g}$ in the twisted chiral multiplets for which the potential energy density given by

$$
\begin{aligned}
U(x, \sigma) & =|D|^{2}+\sum_{\alpha}\left|F_{\alpha}\right|^{2}+\sigma^{\dagger} M(x) \sigma \\
D & =\sum_{\alpha} Q_{\alpha}\left|x_{\alpha}\right|^{2}-\rho \in \mathfrak{g}_{\mathbb{R}}^{*} \\
F_{\alpha} & =\frac{\partial W}{\partial x_{\alpha}} \\
M & =\sum_{\alpha}{ }^{t} Q_{\alpha} Q_{\alpha}\left|x_{\alpha}\right|^{2} \in \mathfrak{g}_{\mathbb{R}}^{\otimes 2}
\end{aligned}
$$

vanishes. Solutions related by the action of the compact real form of the gauge group $G$ via $q$ are identified (the action on $\sigma$ is trivial).

The solution for $x$ here is familiar. Values of $\rho$ for which $M$ has a kernel in the space of solutions to (2.19) lie in cones and form the faces of a fan in $\mathfrak{g}_{\mathbb{R}}^{*}=\mathbb{R}^{r}$, the secondary fan of $\mathscr{A}$. Large cones in this fan are associated to a choice of triangulation of $\mathscr{A}$ which, in turn, 
determines a fan $\Sigma$. This also gives an associated irrelevant ideal $B$ in the homogeneous coordinate ring $S=\mathbb{C}\left[x_{1}, \ldots, x_{n}\right]$. We then get the usual toric variety

$$
Z_{\Sigma}=\frac{\operatorname{Spec} S-V(B)}{G} .
$$

The hyperplane condition (2.17) implies that $Z_{\Sigma}$ is not compact and its canonical divisor $K_{Z_{\Sigma}}$ is zero. However, at this stage, this divisor need not be Cartier and thus $Z_{\Sigma}$ need not be Gorenstein. We do not know a physical reason why one should impose the condition of being Gorenstein on $Z_{\Sigma}$.

For values of $\rho$ deep inside one of these maximal cones, the gauge symmetry is broken at high energies and a semiclassical description is valid. From (2.19) the space of vacua is the critical set of $W$. In general there are massless fields in the low-energy theory interacting via superpotential interactions and the vacuum structure is that of a "bad" hybrid theory. ${ }^{3}$ Suppose this critical set has a non-compact component extending to arbitrarily large expectation values for the fields. Near these solutions semiclassical considerations are valid and the noncompact space supports a continuum of states extending down to zero energy. This leads to singular low-energy behavior [1].

Similarly values of $\rho$ lying in the faces of the secondary fan can also lead to classically singular IR physics. In the gauged linear $\sigma$-model this singularity is evident in that the space of supersymmetric field configurations includes configurations for which $M(x)$ has a kernel. This means a continuous subgroup of $G$ is not broken and the associated $\sigma$ field is free. This introduces a noncompact component into the space of vacua, and again far out along this component semiclassical considerations are a good approximation and we expect the continuous spectrum to extend down to zero energy and lead to singular IR behavior.

These classical considerations are modified by quantum corrections, which we discuss in section 2.6, but in the regions of large $\sigma$ expectation values these are very well controlled. In principle these considerations cannot exclude the possibility that there are intrinsically strongly coupled singularities that are not determined by semiclassical considerations. This can be demonstrated by using the existence of two twisted topological versions of these models, the $\mathbf{A}$ and $\mathbf{B}$ models of [17]. In the topological models semiclassical calculations are exact and lead to the conclusions described in detail in the following two subsections.

We thus arrive at our criterion for a theory being nonsingular. The space of classical vacua given by (2.19) as corrected below must be compact. A generic member of the family will be nonsingular as long as the singular locus is of codimension at least one in $\mathscr{M}_{A}$ and $\mathscr{M}_{B}$.

\subsection{Geometry}

A natural question to ask is when $X$ is a Calabi-Yau variety and so our theory has an interpretation as a nonlinear $\sigma$-model at large radius. This turns out to be a surprisingly awkward question.

The strongest constraint one can impose is that there is a "nef partition". Let $s=$ $\langle\mu, \nu\rangle$. In [18] it was shown that this is equivalent (assuming we are in the reflexive case)

\footnotetext{
${ }^{3}$ That is, a "pseudo-hybrid" in the sense of [16].
} 
to both $\mathscr{A}$ and $\mathscr{B}$ admitting a "special $(s-1)$-simplex". That is, there is an $(s-1)$ simplex with its $s$ vertices in $\mathscr{A}$ such that each facet of Conv $\mathscr{A}$ contains $s-1$ vertices of the simplex; and similarly for $\mathscr{B}$. There is then a triangulation, $\Sigma$, of $\mathscr{A}$ such that each simplex in the triangulation has the special $(s-1)$-simplex as a face. Then $X_{\Sigma}$ will be a complete intersection of $s$ hypersurfaces and a Calabi-Yau variety. Furthermore, the mirror of $X_{\Sigma}$ will also be a complete intersection Calabi-Yau.

Dropping this nef partition condition, we could still have $\mathscr{A}$ admits a special $(s-1)$ simplex but $\mathscr{B}$ doesn't. Then $\mathscr{A}$ could still have a triangulation leading to a Calabi-Yau complete intersection but its mirror does not. An example of this is the Z-manifold [8].

Going further, it might be that neither $\mathscr{A}$ nor $\mathscr{B}$ admit a special $(s-1)$-simplex. Then there is no "obvious" geometric phase but there are still ways that $X$ could really be geometric. For example

1. $Z$ contains more than one component in its compact part, like an exoflop, but Crit $(W)$ misses all but one of these components. Thus $X_{\Sigma}$ looks like a complete intersection again even though the triangulation $\Sigma$ is not associated to a $(s-1)$-simplex.

2. There could be a large radius limit point in $\mathscr{M}_{A}$ that is not a phase limit point. Such cases, associated to flops, were discussed in [19, 20]. More general cases are presumably possible. In particular, large radius limits can hide on the singular locus deep inside the moduli space, away from phase limits.

Note that we can always force a geometric interpretation by including $\nu$ as an extra ray in the fan $\Sigma$. If $\nu$ is interior to $\mathscr{A}$ this puts a divisor in the new $Z_{\Sigma}$ and our new quasi- $X_{\Sigma}$ will be a variety of dimension $d-2$. If $s>1$ it will not be Calabi-Yau. Such a construction is common in the literature (starting with the cubic sevenfold of [21] as the mirror of the Z-manifold) but we want to claim it is unnatural in the context of the gauged linear $\sigma$-model. ${ }^{4}$ Indeed, the extremal transitions between geometric and non-geometric models in section 3.2 will show no signs of this $(d-2)$-fold.

\subsection{The GKZ Determinant for $\mathscr{B}$}

\subsubsection{The generic $\mathbb{C}^{*}$ orbit}

Let us consider first the family of complex structures parametrized by $\mathscr{M}_{B}$. The singular locus in $\mathscr{M}_{B}$ is not subject to quantum corrections and can be determined directly from the space of classical vacua. For generic values of the complexified FI parameters, supersymmetric field configurations will be given by an assignment of constant expectation values to the scalars in the chiral multiplets $x$, identified up to the action of $G$.

Within $Z_{\Sigma}$ we have the classical vacuum space $X_{\Sigma}$ given by the critical locus of $W$. When $X_{\Sigma}$ is non-compact the model is singular. The choice of superpotential for which the theory is singular should not depend at all on the choice of twisted chiral parameters $\tau$, so we should not need to choose a fan $\Sigma$.

\footnotetext{
${ }^{4}$ Not that it is without mathematical virtue. For example, the Calabi-Yau category of $X$ appears as a factor in the semiorthogonal decomposition of the derived category of the $(d-2)$-fold. See section 3.2.
} 
The torus $T_{N}$ acts on $Z_{\Sigma}$ but does not generally fix $X_{\Sigma}$. However, because of the $R$-symmetry, we know that the one-parameter subgroup $\lambda^{\nu}: \mathbb{C}^{*} \rightarrow T_{N}$ given by $\nu \in N$ will preserve $X_{\Sigma}$. We can use the cone-orbit correspondence [13] to see how $\lambda^{\nu}\left(\mathbb{C}^{*}\right)$ acts on $Z_{\Sigma}$. In particular, suppose $x \in X_{\Sigma}$ is a point whose homogeneous coordinates are all nonzero. Then the cone-orbit correspondence tells us that

$$
\lim _{t \rightarrow 0} \lambda^{\nu}(t) x
$$

lies within $X_{\Sigma}$ if and only if $\nu$ lies in the fan $\Sigma$. Similarly

$$
\lim _{t \rightarrow \infty} \lambda^{\nu}(t) x
$$

lies within $X_{\Sigma}$ if and only if $-\nu$ lies in the fan $\Sigma$. But the hyperplane condition for $\mathscr{A}$ means that $\nu$ and $-\nu$ cannot both be in $\Sigma$ and so the orbit $\lambda^{\nu}\left(\mathbb{C}^{*}\right)$ cannot be compact.

Thus we have

Proposition 1. If $X_{\Sigma}$ contains a point with all homogeneous coordinates nonzero then $X_{\Sigma}$ cannot be compact and the associated gauged linear $\sigma$-model is singular.

GKZ [10] have defined a discriminant which exactly determines when this happens. Let $z_{1}, \ldots, z_{d}$ denote affine coordinates on $Z_{\Sigma}$ which may be constructed as Laurent monomials in the homogeneous coordinates using the matrix $A$. Then $W$ may be written as a Laurent polynomial in $z_{j}$ 's. Let $\nabla_{0} \in \mathbb{C}^{\mathscr{B}}$ denote the set of all $W$ for which there exists a point with nonzero $z_{j}$ 's such that

$$
\frac{\partial W}{\partial z_{j}}=0, \quad \forall j
$$

and let $\nabla_{\mathscr{B}}$ denote the closure. (The homogeneity of $W$, forced by the $R$-symmetry, will imply $W=0$ automatically.) Assuming $\nabla_{\mathscr{B}} \subset \mathbb{C}^{\mathscr{B}}$ is codimension one, this defines a

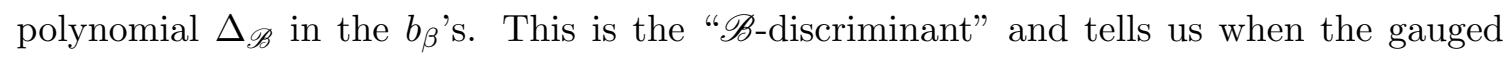
linear $\sigma$-model becomes singular because of the above $\mathbb{C}^{*}$-orbit.

Using the Horn uniformization of [10] and, as we will discuss more carefully in section 2.6.1, we can use the fact that $\mathscr{M}_{B}$ is (possibly redundantly) parametrized by $b_{\beta}$ up to rescalings of the $x_{\alpha}$ to state this condition more directly in terms of the coefficients. The gauged linear $\sigma$-model will be singular for any values of $b_{\beta}$ equivalent up to rescaling to the solution of

$$
\sum_{\beta \in \mathscr{B}}\langle\beta, \alpha\rangle b_{\beta}=0 \quad \forall \alpha \in \mathscr{A}
$$

\subsubsection{Special orbits}

But this is not the only way to find singularities. We should also consider setting some of the $x_{\alpha}$ 's equal to zero, thus killing some of the terms in the superpotential. Consider a subset of coordinates we wish to set to zero. This corresponds to a subset of the pointset $\mathscr{A}$. If the rays through these points do not span a cone, $\tau$, then we cannot simultaneously set these coordinates to zero. 
Assuming $\tau$ exists, we restrict to the subvariety corresponding to the zeroes of these coordinates by quotienting $N_{\mathbb{R}}$ by the span of the rays through these points. The sub-toric variety is then determined by the fan given by $\operatorname{Star}(\tau)$ (see proposition 3.2 of [13]).

If the fan $\operatorname{Star}(\tau)$ is complete we have a compact toric subvariety and so we need not concern ourselves with the possibility of decompactifying the classical vacuum $\operatorname{Crit}(W)$. In this case, the $\mathbb{C}^{*}$ action given by the R-symmetry can have nontrivial orbits which are compact.

The case that we still need to worry about is when $\tau$ lives in a face of the convex hull of the pointset $\mathscr{A}, \operatorname{Conv} \mathscr{A}$. It is then that $\operatorname{Star}(\tau)$ is not complete.

So let $\Upsilon$ denote a face of Conv $\mathscr{A}$ and let $\Upsilon^{\perp}$ denote the points in $M_{\mathbb{R}}=M \otimes_{\mathbb{Z}} \mathbb{R}$ perpendicular to this. Then, upon setting the homogeneous coordinates in the face $\Upsilon$ to zero, the only terms in the superpotential which remain nonzero correspond to $\mathscr{B} \cap \Upsilon^{\perp}$. Subsets of $\mathscr{A}$ which do not correspond to faces of Conv $\mathscr{A}$ do not yield any other possibilities.

Let Cone $(\text { Conv } \mathscr{A})^{\vee}$ denote the cone in $M_{\mathbb{R}}$ dual to the cone over Conv $\mathscr{A}$. We have just shown that an interesting superpotential to consider is one where the nonzero monomials lie in a face of Conv $\mathscr{B}$ which lies in the boundary of $\operatorname{Conv}^{\vee} \mathscr{A}$. Let $\Gamma$ denote the such a face of Conv $\mathscr{B}$. Then noncompact orbits of the R-symmetry $\mathbb{C}^{*}$ action can occur only if the discriminant $\Delta_{\mathscr{B} \cap \Gamma}$ vanishes.

This is getting very close to reproducing the GKZ " $\mathscr{B}$-determinant". This is defined as [10] as resultant polynomial, $E_{\mathscr{B}}$, associated to the simultaneous vanishing of

$$
x_{1} \frac{\partial W}{\partial x_{1}}, x_{2} \frac{\partial W}{\partial x_{2}}, \ldots, x_{n} \frac{\partial W}{\partial x_{n}} .
$$

It is shown that $[10]$

$$
E_{\mathscr{B}}=\prod_{\Gamma} \Delta_{\mathscr{B} \cap \Gamma}^{u(\Gamma)}
$$

where the product is over the faces of Conv $\mathscr{B}$. To be precise, faces are included by the following rules starting from faces of dimension zero and working upwards:

1. All vertices are included.

2. Higher dimensional faces are included only when they add points obeying nontrivial affine relations.

For example, if Conv $\mathscr{B}$ were a simplex and only had points at the vertices, only the vertices would be included in the product (2.26). Higher dimensional faces appear in the product if they are non-simplicial or have internal points. The multiplicity $u(\Gamma)$ is a positive integer which, while potentially very interesting, will not concern us in this paper. We show some simple examples in figure 1.

Definition 2. The pointsets $\mathscr{A}$ and $\mathscr{B}$ are reflexive if

$$
\operatorname{Cone}(\operatorname{Conv} \mathscr{A})^{\vee}=\operatorname{Cone}(\operatorname{Conv} \mathscr{B}) \text {. }
$$




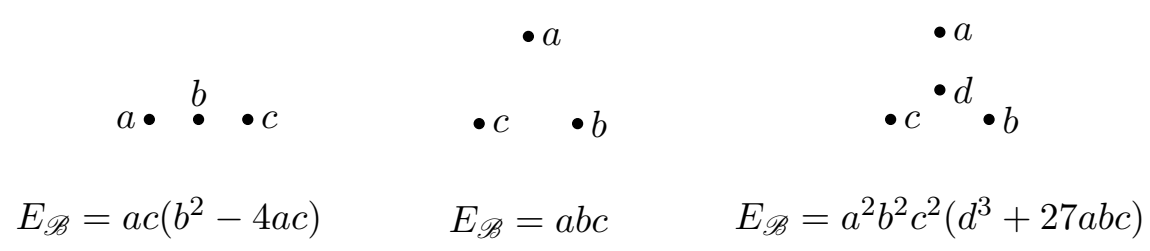

Figure 1. Some examples of $E_{\mathscr{B}}$ for a given $\mathscr{B}$.
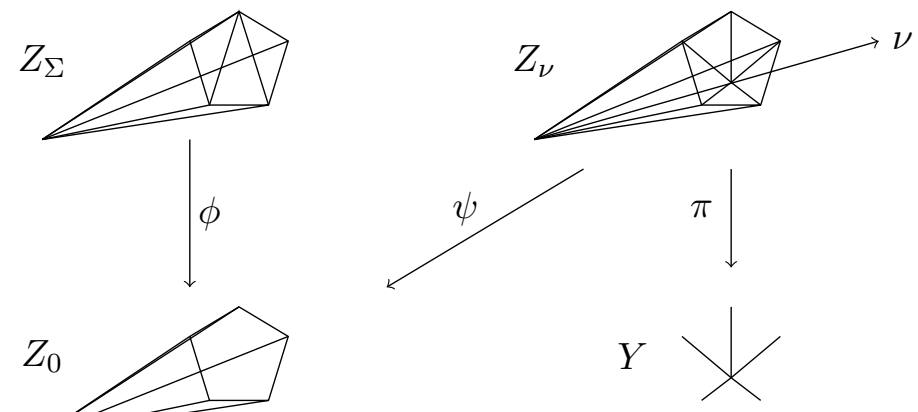

Figure 2. Crit $(W)$ is compact if it is fixed by the R-action.

In this case there is a correspondence between faces of Conv $\mathscr{A}$ and dual faces of Conv $\mathscr{B}$. So the product over the faces of Conv $\mathscr{B}$ in (2.26) corresponds to a product over faces of Conv $\mathscr{A}$, i.e., setting various combinations of $x_{\alpha}$ 's to zero. Thus, if $E_{\mathscr{B}}$ is nonzero then the $\mathbb{C}^{*}$ orbits acting within $\operatorname{Crit}(W)$ associated to the R-symmetry will be compact.

We now invoke a key result from GKZ. Namely we use proposition 1.1 of chapter 10 of [10], which can be stated as

Proposition 3. If $M_{\mathbb{R}}$ is generated $d^{5}$ by $\mathscr{B}$ then the variety determined by simultaneous vanishing of (2.25) is codimension one and corresponds to the determinant function $E_{\mathscr{B}}$. This determinant is generically nonzero.

The reflexive condition forces $\mathscr{A}$ to generate $M_{\mathbb{R}}$ and so we have

Proposition 4. If the pointsets $\mathscr{A}$ and $\mathscr{B}$ are reflexive then the $\mathbb{C}^{*}$ orbits acting within $\operatorname{Crit}(W)$ associated to the $R$-symmetry will be compact for generic values of the coefficients $b_{\beta}$ in the superpotential.

\subsubsection{A sufficient condition for compactness}

Any time we have a nontrivial R-symmetry $\mathbb{C}^{*}$ orbit, the theory is noncompact and thus singular. We would now like to prove that the only way that the gauged linear $\sigma$-model can become singular is when we have such orbits. That is, if $\operatorname{Crit}(W)$ is fixed by the R-symmetry, we need to show it is compact.

\footnotetext{
${ }^{5} \mathrm{GKZ}$ assume the stronger condition that $\mathscr{B}$ affinely generates $M \cap H_{\nu}$. This is the same as our assumption up to a torsion group but that is not important for the statement that the determinant is generically nonzero.
} 
To do this we consider various maps as shown in figure 2. Let $Z_{0}$ be the toric variety associated to the cone over $\operatorname{Conv}(\mathscr{A})$. We therefore have a toric map $\phi: Z_{\Sigma} \rightarrow Z_{0}$, which contracts various toric subspaces.

It is clear that reflexive condition forces $\nu$ to lie in the proper interior the $\operatorname{Cone}(\operatorname{Conv}(\mathscr{A}))$. This means we can consider another toric variety $Z_{\nu}$ obtained from a star subdivision along the ray passing through $\nu$ as shown in figure 2. This gives a toric map $\psi: Z_{\nu} \rightarrow Z_{0}$ contracting the divisor associated to $\nu$.

Consider the $\mathbb{C}^{*}$-action associated to $\nu \in N$. This extends to a well-behaved $\mathbb{C}$-action on $Z_{\nu}$. Quotient $Z_{\nu}$ by this $\mathbb{C}$ by projecting the lattice $N$ onto the quotient lattice $N / \nu$. This projects the fan to produce a new toric variety $Y$. The resulting map

$$
\pi: Z_{\nu} \rightarrow Y
$$

is again toric. Because $\nu$ was in the interior of $\operatorname{Cone}(\operatorname{Conv}(\mathscr{A}))$, the resulting fan is complete and thus $Y$ is compact. Now $\operatorname{Crit}(W)$ is an algebraic subset and thus closed. Therefore its image in the quotient $Y$ is compact. Furthermore, the map $\phi$ is proper from theorem 3.4.11 of [13]. This means the inverse image of a compact set in $Z_{0}$ is compact in $Z_{\Sigma}$. It follows that if $\operatorname{Crit}(W)$ has only compact orbits under the R-symmetry $\mathbb{C}^{*}$-action, by chasing the maps in figure 2 , it must be compact in $Z_{\Sigma}$.

We have therefore proven:

Proposition 5. For the fields so far considered, for a generic choice of coefficients, $b_{\beta}$, the gauged linear $\sigma$-model is nonsingular if the pointsets $\mathscr{A}$ and $\mathscr{B}$ are reflexive.

The singular models are contained in the locus $E_{\mathscr{B}}=0$. As we will see, however, this condition is too strong in general, and there are typically many smooth models for which $E_{\mathscr{B}}=0$. Furthermore $\mathscr{A}$ and $\mathscr{B}$ do not need to be reflexive to give a generically smooth model.

Note that the previous motivation for reflexivity in $[4,5]$ was that $Z_{\Sigma}$ should be a vector bundle over a Gorenstein variety. $X_{\Sigma}$ (assuming it is geometric) is then viewed as a complete intersection in the Gorenstein variety.

\subsection{The determinant for $\mathscr{A}$}

\subsubsection{The primary component}

The considerations of the previous section determined conditions under which the family of gauged linear $\sigma$-models includes sufficiently general superpotentials that the generic member will not be singular, assuming that $Z_{\Sigma}$ is sufficiently generic. A key observation was that the singular locus in $\mathscr{M}_{B}$ is independent of the twisted chiral parameters. In this section our goal is to understand the singular locus in $\mathscr{M}_{A}$ and the combinatorial conditions under which the generic element of the family avoids this. As mentioned above, a tree level analysis finds singular IR limits and a non-compact space of vacua for values of $\rho$ lying in the faces of the secondary fan.

These classical expectations are corrected by quantum effects as found in $[1,3]$. These introduce a dependence on the periodic $\theta$ angles for the gauge group. The IR physics 
in fact depends holomorphically on the combination $q_{j}=e^{2 \pi i \tau_{j}}$. The scalar fields $\sigma$ are contained in twisted chiral multiplets and their interactions controlled by a holomorphic twisted superpotential whose critical points are the vacua. The classical superpotential

$$
\widetilde{W}_{0}=\sum_{j=1}^{r} \Sigma_{j} \log q_{j}
$$

leads to the cone structure described above, and we can now see how quantum effects modify this.

The noncompact space of vacua responsible for the singular low-energy physics lie at large values of $\sigma$. If $\sigma$ is nonzero then (2.19) can lead to mass terms for the chiral fields. Let us suppose, for the time being, that $\sigma$ takes a value that is sufficiently generic so that all the $x_{\alpha}$ 's become massive. We deal with the more general case in section 2.6.2. Integrate out these massive fields to produce a correction to the twisted superpotential

$$
\widetilde{W}_{1}=-\sum_{j=1}^{r} \Sigma_{j}\left[\sum_{\alpha=1}^{n} Q_{\alpha}^{j} \log \left(\sum_{k=1}^{r} Q_{\alpha}^{k} \Sigma_{k}\right)\right] .
$$

Critical points of the twisted superpotential $\widetilde{W}_{0}+\widetilde{W}_{1}$ satisfy

$$
q_{j}=\prod_{\alpha}\left(\sum_{k} Q_{\alpha}^{k} \sigma_{k}\right)^{Q_{\alpha}^{j}} .
$$

These are a set of $r$ homogeneous equations for the $r$ expectation values $\sigma_{j}$. For generic $q_{j}$ there will be no solutions, while for values of $q_{j}$ for which a solution exists there will be a noncompact flat direction for $\sigma$. We can consider these as parametric equations determining values of $q_{j}$ for which the model is singular. This exactly reproduces the "Horn uniformization" of equation (3.3) in for the $A$-discriminant in [10]. If this singular set is codimension one (as it is in most examples) we call it the "primary component" of the singular locus.

To compare this to the expressions from the previous section it is helpful to express the parameters $q_{j}$ in terms of a redundant set $a_{\alpha}$ as

$$
q_{j}=\prod_{\alpha} a_{\alpha}^{Q_{\alpha}^{j}},
$$

expressing them as affine coordinates on a quotient of $\mathbb{C}^{n}$ by $\left(\mathbb{C}^{*}\right)^{d}$. In terms of the $a_{\alpha}$ we see that the solutions to $(2.31)$ are precisely the orbits under this action containing a solution to

$$
a_{\alpha}=\sum_{k} Q_{\alpha}^{b} \sigma_{k}
$$

The sequence (2.3) shows that this is equivalent to

$$
\sum_{\alpha \in \mathscr{A}}\langle\beta, \alpha\rangle a_{\alpha}=0 \quad \forall \beta \in \mathscr{B} .
$$

The locus parametrized by (2.31) has large $-|\rho|$ limits which asymptote, up to a finite shift, to some of the faces of the secondary fan. This is expected in the sense that in these 
limits the quantum corrections are suppressed. In these limits the solution for $\sigma$ lies in the direction of the symmetry that is unbroken in the classical calculation. When $\sigma$ points in these special directions, (2.19) leaves a subset of the chiral fields $x_{\alpha}: Q_{\alpha}^{j} \sigma_{j}=0$ massless. In the interior of $\rho$-space, the true singular locus interpolates smoothly between these various limits. At a generic point on it the $\sigma$ vacua are in a generic direction in $\sigma$-space leading to nonzero masses for all of the chiral fields. These singularities are insensitive to the superpotential, since the chiral fields are massive.

\subsubsection{Other components}

So far we have considered vacua where either $x$ 's are interesting and the $\sigma$ 's are all zero, i.e., Higgs branches; or vacua where the $\sigma$ 's are nonzero and the $x$ 's are all massive, i.e., Coulomb branches. Now consider the mixed cases where the $\sigma$ fields can be nonzero but some $x$ 's remain massless. This will lead us to looking at faces of Conv $\mathscr{A}$ as we now explain.

To understand these other components we first ignore the superpotential and seek loci in which there are such mixed branches at $W=0$.

Choose a subgroup $\widehat{G}_{h} \subset \widehat{G}$ and let $\widehat{G}_{c}$ be the quotient $\widehat{G} / \widehat{G}_{h}$, with $\pi_{h}$ the quotient map. Let $e_{\alpha}$ be a basis for $\mathbb{Z}^{\mathscr{A}}$. Then define

$$
\begin{aligned}
& \mathscr{A}_{h}=\left\{\alpha \in \mathscr{A}: \pi_{h} Q e_{\alpha}=0\right\} \\
& \mathscr{A}_{c}=\mathscr{A}-\mathscr{A}_{h} .
\end{aligned}
$$

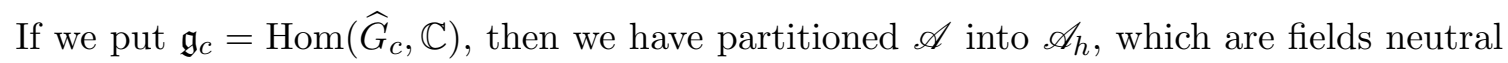
under $\mathfrak{g}_{c}$; and $\mathscr{A}_{c}$, which are charged.

Proposition 6. There is a commutative diagram

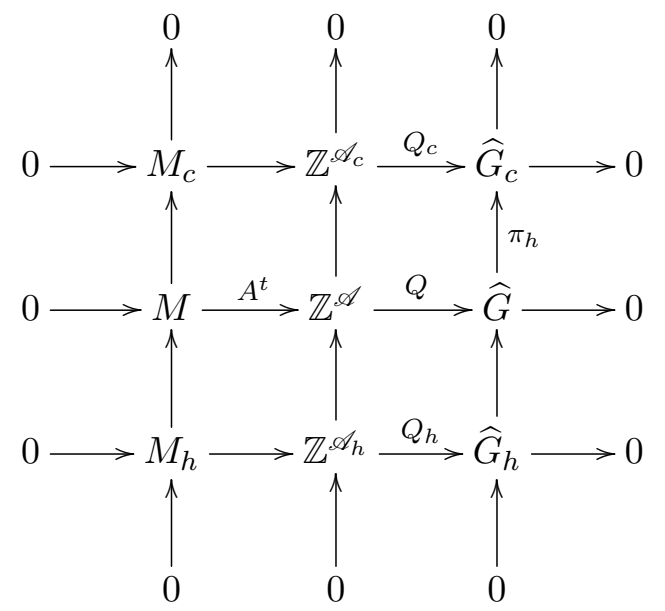

with all rows and columns exact. Here $Q_{c}$ is defined as $\pi_{h} Q$ restricted to $\mathbb{Z}^{\mathscr{A}_{c}}$, and $Q_{h}$ exists uniquely to make the diagram commute. $M_{c}$ and $M_{h}$ are defined as the kernels of $Q_{c}$ and $Q_{h}$ respectively.

To prove this proposition note that the first two columns consist of free groups; then use projectivity to prove the existence of the maps. Then use the Snake Lemma to prove the first column is exact. 
Now assume that $\sigma$ acquires a large generic value in $\mathfrak{g}_{c}$, which generates masses for all the chiral fields in $\mathscr{A}_{c}$. Integrating out these fields associated to $\mathscr{A}_{c}$ will produce an effective twisted superpotential for $\sigma$ and a correction to the $D$-terms in $\mathfrak{g}_{c}$. The $D$-terms for $\mathfrak{g}_{h}$ will not be corrected. The resulting model decomposes into a "product" of a twisted Landau-Ginzburg theory for $\sigma \in \mathfrak{g}_{c}$ and a reduced gauged linear $\sigma$-model involving the chiral fields associated to points in $\mathscr{A}_{h}$ and the reduced gauge group $G_{h}$.

So we have a mixed Higgs-Coulomb branch where the points in $\mathscr{A}_{h}$ are associated to the Higgs data and the points in $\mathscr{A}_{c}$ are associated to the Coulomb data. The primary component in the previous section corresponds to the case $\widehat{G}_{h}=\widehat{G}$; i.e., $\mathscr{A}_{h}=\emptyset$ and $\mathscr{A}_{c}=\mathscr{A}$.

The Landau-Ginzburg model for the Coulomb branch will have supersymmetric vacua, hence flat directions leading to singular IR physics, when the FI parameters in $\mathfrak{g}_{c}^{*}$ satisfy

$$
q_{j}=\prod_{\alpha \in \mathscr{A}_{c}}\left(\sum_{k} Q_{c, \alpha}^{k} \sigma_{k}\right)^{Q_{c, \alpha}^{j}} .
$$

We have an exact sequence

$$
0 \longrightarrow \mathfrak{g}_{h}^{*} \longrightarrow \mathfrak{g}^{*} \longrightarrow \mathfrak{g}_{c}^{*} \longrightarrow 0,
$$

so we have a singular theory if the Kähler parameters of the total theory lie in the inverse image of the Kähler parameters of the Coulomb theory satisfying (2.37).

Now we turn our attention to the gauged linear $\sigma$-model associated to the Higgs data. The points $\mathscr{A}_{h}$ should be viewed as lying in the quotient lattice $N_{h}=N / N_{c}$. The first thing to observe is that this generally destroys the Calabi-Yau condition - the points will not lie in a hyperplane, and so we should take care to see where the IR flow lands us. Let us build the secondary fan $F_{h}$ for the Higgs data $\mathscr{A}_{h} \subset N_{h}$ in the usual way (see, for example, section 15.2 of [13]). There is no reason the suppose this fan is complete. The case of an incomplete fan for non-Calabi-Yau data is familiar from [1, 3]. Here the IR flow pushes us beyond the exterior walls of the fan to where supersymmetry is naïvely broken. More careful analysis shows that supersymmetry is unbroken, but there are nonzero expectation values for some of the $\sigma$ fields living in $\mathfrak{g}_{h}$. So, in this case, our model is just reproducing a different Coulomb-Higgs split where $\mathscr{A}_{c}$ is larger.

To avoid reproducing multiple copies of the same components of the discriminant we should restrict attention to case where the secondary fan $F_{h}$ is complete. Now the region covered by the secondary fan is spanned by positive combinations of columns of $Q_{h}$. Let $\mathbf{x}_{\alpha}$ be the position vector of the point $\alpha \in \mathscr{A}_{h}$ in $N_{h}$. So the fan cannot be complete if there is any nontrivial relationship

$$
\sum_{\alpha \in \mathscr{A}_{h}} Q_{\alpha} \mathbf{x}_{\alpha}=0
$$

where all the $Q_{\alpha}$ 's are $\geq 0$. This imposes two conditions:

1. No point in $\mathscr{A}_{h} \subset N_{h}$ should lie at the origin. $\mathscr{A}_{c}$ spans the subspace $N_{c, \mathbb{R}} \subset N_{\mathbb{R}}$ and so we require $\mathscr{A}_{h} \cap N_{c, \mathbb{R}}=\emptyset$. That is, $\mathscr{A}_{c}=N_{c, \mathbb{R}} \cap \mathscr{A}$. 
2. The subspace $N_{c, \mathbb{R}}$ must meet Conv $\mathscr{A}$ along a face.

We have therefore almost recovered the GKZ prescription of considering all the faces of the convex hull of $\mathscr{A}$ similar to (2.26). There is a notable difference, however, when we ask exactly which faces are included. We include faces given by $\mathscr{A}_{c}$ when, and only when, there is a $\mathfrak{g}_{c}$ such that $\mathscr{A}_{c}$ is the set of fields with nonzero charges. Working upwards from lower dimensions, these are precisely the faces with a new nontrivial affine relation. We therefore reproduce the GKZ rules of section 2.5.2, except we have no vertices in the product. We return to this issue shortly.

Anyway, we can use the results of GKZ again to prove that the model is nonsingular for a generic choice of Kähler data so long as $\mathscr{A}$ generates $N_{\mathbb{R}}$. So we only need modify proposition 5 slightly to obtain:

Theorem 7. For a generic choice of coefficients, $b_{\beta}$, and values of $i \rho_{j}+\frac{\theta_{j}}{2 \pi}$, the gauged linear $\sigma$-model is nonsingular if the pointsets $\mathscr{A}$ and $\mathscr{B}$ are reflexive.

\subsection{Mirror symmetry}

There is an obvious symmetry in the above construction that is, of course, mirror symmetry:

$$
\begin{aligned}
N & \leftrightarrow M \\
\nu & \leftrightarrow \mu \\
\mathscr{A} & \leftrightarrow \mathscr{B} \\
a_{\alpha} & \leftrightarrow b_{\beta} \\
\mathscr{M}_{A} & \leftrightarrow \mathscr{M}_{B}
\end{aligned}
$$

Let $Y$ refer to the mirror of $X$ when the above mirror switch is applied.

In particular, the last of the identifications in (2.40) is an very interesting pointwise map between our models of the Kähler moduli space of $X$ and then complex structure moduli space of $Y$ (and vice versa). It was first proposed in [3] and is a natural extension of the "monomial-divisor mirror map" of [22]. Asymptotically, in a large radius limit of $X$ and near a point of maximally unipotent monodromy, it becomes the usual mirror map. However, the mirror map of (2.40) is a global identification over the whole parameter space and is less well studied. In [3] this identification was proved for models describing hypersurfaces in simplicial toric varieties. For the more general case considered here it is a natural conjecture. In particular, of course, this implies that mirror symmetry respects the restriction of the full moduli spaces to the toric subspaces. This is known to be true when we have dual nef-partitions of reflexive polytopes $[4,5]$.

Our analysis above does not quite respect this mirror symmetry. The GKZ determinant corresponding to decompactification of $x$ vacua is not mirror to the condition corresponding to decompactification of $\sigma$ vacua. In particular, we included points at vertices of Conv $\mathscr{B}$ but we ignored the points at vertices of Conv $\mathscr{A}$. We need to resolve this apparent violation of mirror symmetry.

The contribution of a vertex point in $\mathscr{B}$ to $E_{\mathscr{B}}$ is simply the associated coefficient $b_{\beta}$ (perhaps raised to some power). So the nonsingularity is associated to setting $b_{\beta}=0$. For 
mirror symmetry to be respected, we need some kind of singularity when $a_{\alpha}=0$, if $\alpha$ is a vertex point.

We can put a metric on $Z_{\Sigma}$ using the moment map in $\mathfrak{g}_{\mathbb{R}}^{*}$ which is given by the values of the $\rho_{\alpha}$ 's. Following [22] we have

Proposition 8. The particular triangulation we use for $\mathscr{A}$ is the one consistent with a convex piecewise linear function taking the values of a "height function" $\log \left|a_{\alpha}\right|$ at each point $\alpha \in \mathscr{A}$.

Thus, the mirror to $b_{\beta}=0$ is setting the height function of $\alpha$ to $-\infty$. This results in a component of $Z_{\Sigma}$ becoming infinitely large. Thus $X_{\Sigma}$ may decompactify.

The way the vertices of Conv $\mathscr{A}$ contribute to the discriminant is therefore quite different to the other faces. The non-vertex faces are associated to singularities induced by noncompactness of the Coulomb branch while the vertices correspond to noncompactness of the Higgs branch. For Conv $\mathscr{B}$, all the faces, whether vertices are not, give singularities associated to noncompactness of the Higgs branch.

\subsection{Extremal transitions and breaking the reflexive condition}

Consider the left-most example in figure 1. One might expect to produce the discriminant of the familiar quadratic equation $a x^{2}+b x+c$. But we get $a c\left(b^{2}-4 a c\right)$ rather than $b^{2}-4 a c$. Essentially, this is because $E_{\beta}$ looks for solutions of the weaker equations $x_{i} \frac{\partial W}{\partial x_{i}}=0$ where $W=a x_{0}^{2}+b x_{0} x_{1}+c x_{2}^{2}$. As such, $E_{\beta}=0$ is a stronger condition than non-compactness of Crit $W$. This means that the conditions we considered above may well be too strong, and indeed they are. That is, in some cases we do not require points in $\mathscr{B}$ at the vertices of the dual cone of Conv $\mathscr{A}$.

Mirror to this should be the statement that we can sometimes drop points at the vertices of Conv $\mathscr{A}$. Above we argued that dropping such points amounts to a limit in Kähler moduli space where a previously compact subset in $Z_{\Sigma}$ becomes infinitely large. This need not cause problems with the gauged linear $\sigma$-model since $\operatorname{Crit}(W)$ may miss this infinitely large component.

It turns out that by considering extremal transitions we are forced to consider nonreflexive pairs. First consider the following:

Definition 9. The pointsets $(\mathscr{A}, \mathscr{B})$ are $\mathscr{B}$-complete if

$$
\operatorname{Conv}\left(\operatorname{Cone}(\operatorname{Conv} \mathscr{A})^{\vee} \cap M \cap H_{\nu}\right)=\operatorname{Conv}(\mathscr{B}),
$$

and similarly $\mathscr{A}$-complete if

$$
\operatorname{Conv}\left(\operatorname{Cone}(\operatorname{Conv} \mathscr{B})^{\vee} \cap N \cap H_{\mu}\right)=\operatorname{Conv}(\mathscr{A}),
$$

Begin with pointsets $\mathscr{A}$ and $\mathscr{B}$ that correspond to a generically good theory (e.g., they are reflexive). Now remove a point or points from $\mathscr{A}$ to shrink the convex hull. Thus Cone $(\text { Conv } \mathscr{A})^{\vee}$ grows. We can then add points to $\mathscr{B}$ so that $(\mathscr{A}, \mathscr{B})$ is $\mathscr{B}$-complete. This process corresponds (if it is geometric) to an extremal transition as we now review. 

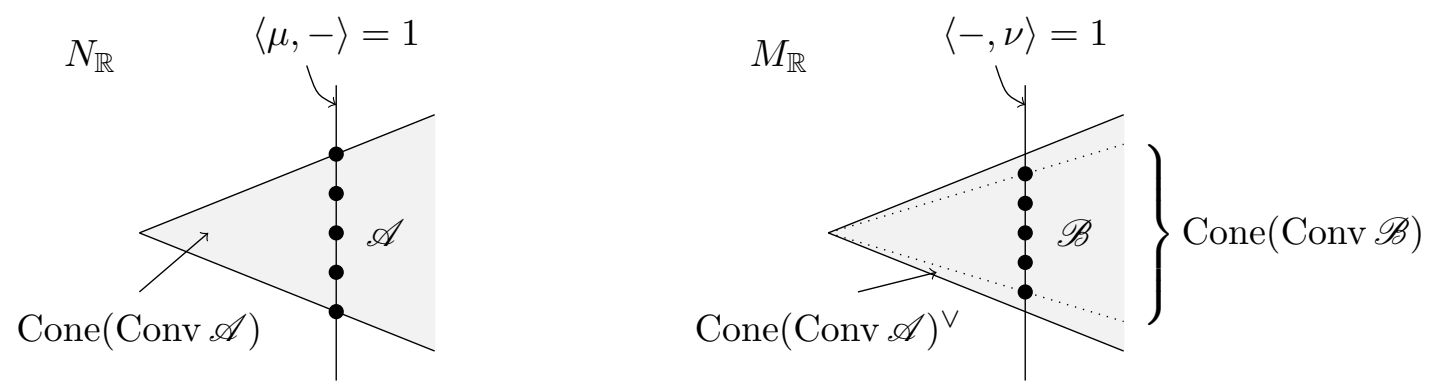

Figure 3. $\mathscr{B}$-Completeness.

Obviously ignoring a point $\beta \in \mathscr{B}$ amounts to setting $b_{\beta}=0$. Thus, by mirror symmetry, we assume the same is true for points in $\mathscr{A}$. We therefore ignore $\alpha \in \mathscr{A}$ by setting $a_{\alpha} \rightarrow 0$, i.e., the height function in proposition 8 to $-\infty$. This picks out a certain class of triangulations of $\mathscr{A}$. If the phase has a geometrical interpretation, it will typically be an "exoflop" phase [20]. This has a line or surface "sticking out" of a singular Calabi-Yau component. We ignore the massless D-branes by ignoring this extra component. Then we smooth the remaining Calabi-Yau component by a deformation of complex structure, by adding to $W$ new allowed monomials, corresponding to the new points in $\mathscr{B}$, to complete the extremal transition.

Given our new enlarged pointset $\mathscr{B}$ we should check to see if there are any possible blow-ups by demanding $\mathscr{A}$-completeness. So we start with a model which is generically smooth and then go via an extremal transition to a new theory. Since we have done all we can to make this new theory smooth, as we argue shortly, it should be smooth! This then gives the conjecture:

Conjecture 10. If a gauged linear $\sigma$-model is defined by pointsets $\mathscr{A}$ and $\mathscr{B}$ which are both $\mathscr{A}$-complete and $\mathscr{B}$-complete then this model is nonsingular for generic values of the complex structure and Kähler parameters.

Note that if the pointsets are reflexive then they satisfy the condition in the conjecture. We show schematically in figure 3 how one can satisfy $\mathscr{B}$-completeness without reflexivity.

From the perspective of string theory compactifications, a singular conformal field theory corresponds to massless D-branes [23]. Given such a string compactification one should be free to deform it to give mass to the D-branes. In this sense, every singular compactification should be infinitesimally close to a nonsingular one. A counterexample to the above conjecture would, naïvely, contradict this idea. This counterexample, with generic values for $a_{\alpha}$ and $b_{\beta}$ would require us to travel a properly nonzero distance to undo the extremal transition to return to a good theory.

Unfortunately we can't claim to have proven this conjecture by such an argument. There are non-toric deformations that are not expressible in terms of varying the parameters $a_{\alpha}$ and $b_{\beta}$. That is, our gauged linear $\sigma$-model only parametrizes a "toric" sub-moduli space of the full moduli space of theories. It may be possible that this toric sub-moduli space lies within the codimension one subset of singular theories within the moduli space. We have 
proven this never happens in the reflexive case so it might be not unreasonable to expect the same in general. This would justify the conjecture.

In section 4.1.2 we will see that even the above conjecture is too strong. We will give an example of a model that is $\mathscr{B}$-complete but not $\mathscr{A}$-complete and yet manages to be generically nonsingular.

\section{Examples}

\subsection{A non-reflexive example}

We can, at least, give an example of a non-reflexive gauged linear $\sigma$-model that is generically nonsingular. Let $X$ be the intersection of a cubic and quartic in weighted projective space $\mathbb{P}_{\{2,1,1,1,1,1\}}^{5}$. According to the usual prescription $[5,8,18]$, one uses the pointset $\mathscr{A}$ given by the rows of the matrix:

$$
A^{t}=\left(\begin{array}{ccccccc}
1 & 0 & 1 & 0 & 0 & 0 & 0 \\
1 & 0 & 0 & 1 & 0 & 0 & 0 \\
1 & 0 & 0 & 0 & 1 & 0 & 0 \\
0 & 1 & 0 & 0 & 0 & 1 & 0 \\
0 & 1 & 0 & 0 & 0 & 0 & 1 \\
0 & 1 & -2 & -1 & -1 & -1 & -1 \\
1 & 0 & 0 & 0 & 0 & 0 & 0 \\
0 & 1 & 0 & 0 & 0 & 0 & 0
\end{array}\right)
$$

These lie in a hyperplane given by $\mu=(1,1,0,0,0,0,0)$. This example is not reflexive. The dual of the cone over Conv $\mathscr{A}$ is not spanned by points living in a hyperplane. Equivalently, in the language of [5], it is not a nef partition of a reflexive polytope. However, we can define the pointset $\mathscr{B}$ from the rows of

$$
B^{t}=\left(\begin{array}{ccccccc}
0 & 1 & 0 & 0 & 0 & 2 & -1 \\
1 & 0 & -1 & -1 & -1 & 4 & 0 \\
1 & 0 & -1 & -1 & -1 & 0 & 0 \\
1 & 0 & 1 & -1 & -1 & 0 & 0 \\
1 & 0 & -1 & 3 & -1 & 0 & 0 \\
1 & 0 & -1 & -1 & 3 & 0 & 0 \\
1 & 0 & -1 & -1 & -1 & 0 & 4 \\
0 & 1 & 0 & 0 & 0 & -1 & -1 \\
0 & 1 & 1 & 1 & 0 & -1 & -1 \\
0 & 1 & 1 & 0 & 1 & -1 & -1 \\
0 & 1 & 1 & 0 & 0 & 0 & -1 \\
0 & 1 & 1 & 0 & 0 & -1 & 0 \\
0 & 1 & 1 & 0 & 0 & -1 & -1 \\
0 & 1 & 0 & 3 & 0 & -1 & -1 \\
0 & 1 & 0 & 0 & 3 & -1 & -1 \\
0 & 1 & 0 & 0 & 0 & -1 & 2
\end{array}\right)
$$


which lie in a hyperplane given by $\nu=(1,1,0,0,0,0,0)$. This does satisfy the conditions of theorem 7 . Ignoring coefficients, $A^{t} B$ defines the superpotential

$$
\begin{aligned}
W=x_{8}\left(x_{2}^{3}+x_{3}^{3}+x_{4}^{3}+x_{5}^{3}+x_{6}^{3}+x_{1} x_{2}+x_{1} x_{3}\right. & \left.+x_{1} x_{4}+x_{1} x_{5}+x_{1} x_{6}\right) \\
& +x_{7}\left(x_{1}^{2}+x_{2}^{4}+x_{3}^{4}+x_{4}^{4}+x_{5}^{4}+x_{6}^{4}\right),
\end{aligned}
$$

as one would expect for this complete intersection. Note that Conv $\mathscr{B} \cap M$ has 126 points. Including any of these points maintains a good theory and corresponds to including further "interior" monomials in (3.3). This yields a nonsingular CFT since the intersection of a cubic and quartic in $\mathbb{P}_{211111}^{5}$ is generically smooth. Note that $\mathbb{P}_{211111}^{5}$ is not Gorenstein.

It is interesting ask whether $X$ has a geometric mirror. As we discussed in section 2.4, this is not an easy question. Let us look for a large radius phase for the mirror. That is, exchanging the rôles of $\mathscr{A}$ and $\mathscr{B}$, can we find a phase of this model corresponding to a complete intersection in a toric variety? We can look for a special $(s-1)$-simplex. In our case we are looking for a 1-simplex with vertices on any of the 126 points in Conv $\mathscr{B}$ such that each facet of Conv $\mathscr{B}$ contains precisely one vertex of this simplex. In this, case a computer aided search shows that no such 1-simplex exists. Thus, there is no obvious "geometric phase", even though with this many points the number of phases is incalculably large. (The work of [24] puts an upper bound of around $10^{30000}$ on this number.)

\subsection{Extremal transitions}

The motivation for conjecture 10 came from considering extremal transitions. Extremal transitions are usually viewed from the perspective of geometry but we can easily have transitions between non-geometric phases too. We will give several examples of the latter. This will also show how to transition between the non-reflexive example of section 3.1 and reflexive cases. Thus one is forced to consider non-reflexive models if all extremal transitions are followed.

\subsection{1 $\quad \mathbb{P}^{5} \mid 42$}

The intersection of a quartic and quadric in $\mathbb{P}^{5}$ is a Calabi-Yau threefold and is given by reflexive data:

$$
A^{t}=\left(\begin{array}{ccccccc}
1 & 0 & 1 & 0 & 0 & 0 & 0 \\
1 & 0 & 0 & 1 & 0 & 0 & 0 \\
1 & 0 & 0 & 0 & 1 & 0 & 0 \\
1 & 0 & 0 & 0 & 0 & 1 & 0 \\
0 & 1 & 0 & 0 & 0 & 0 & 1 \\
0 & 1 & -1 & -1 & -1 & -1 & -1 \\
1 & 0 & 0 & 0 & 0 & 0 & 0 \\
0 & 1 & 0 & 0 & 0 & 0 & 0
\end{array}\right), B^{t}=\left(\begin{array}{ccccccc}
0 & 1 & 2 & 0 & 0 & 0 & -1 \\
1 & 0 & 3 & -1 & -1 & -1 & 0 \\
1 & 0 & -1 & -1 & -1 & -1 & 0 \\
1 & 0 & -1 & 3 & -1 & -1 & 0 \\
1 & 0 & -1 & -1 & 3 & -1 & 0 \\
1 & 0 & -1 & -1 & -1 & 3 & 0 \\
1 & 0 & -1 & -1 & -1 & -1 & 4 \\
0 & 1 & 0 & 0 & 0 & 0 & -1 \\
0 & 1 & 0 & 2 & 0 & 0 & -1 \\
0 & 1 & 0 & 0 & 2 & 0 & -1 \\
0 & 1 & 0 & 0 & 0 & 2 & -1 \\
0 & 1 & 0 & 0 & 0 & 0 & 1
\end{array}\right)
$$




$$
\mu=\nu=(1,1,0,0,0,0,0)
$$

$\mathscr{A}$ has two triangulations. As promised, one gives $X$ as quadric $\cap$ quartic in $\mathbb{P}^{5}$. The other is a "bad hybrid" Landau-Ginzburg fibration over $\mathbb{P}^{1}$.

Now do an extremal transition by dropping $(1,0,0,0,0,0,0) \in \mathscr{A}$. We restore this to a reflexive pair if we add $(-1,2,1,1,1,1,-2)$ to $\mathscr{B}$. For this new theory the points in $\mathscr{A}$ now form the vertices of a simplex and so this model clearly has only one phase. This is a Landau-Ginzburg phase with superpotential

$$
W=x_{1}^{4}+x_{2}^{4}+x_{3}^{4}+x_{4}^{4}+x_{5}^{4}+x_{6}^{4}+b x_{7}^{2}+\ldots,
$$

where the extremal transitions "joins on" at $b=0$. That is, the monomial $x_{7}^{2}$ is the extra point we add to $\mathscr{B}$. Note that, as usual, there is a finite group remnant of $G$ which gives us a Landau-Ginzburg orbifold theory. In this case we have $\mathbb{Z}_{4}$ group acting.

Note that when $b \neq 0$ we are allowed to ignore the massive $x_{7}$. This yields the Gepner model $\mathbf{2}^{6}$. The resulting model effectively has $h^{1,1}=0$ and is clearly not geometric.

It is interesting to picture physically what has happened in this transition. We started with a geometry Calabi-Yau threefold $X$ and then deformed the Kähler form to shrink it down. The resulting type of phase limit was analyzed in [16]. This limit is a hybrid model over the weighted projective space $\mathbb{P}_{\{2,4\}}^{1}$ with fiber a Landau-Ginzburg model. The theory is at finite distance in moduli space and singular. This "badness" of the hybrid model comes from the fact that the base $\mathbb{P}_{\{2,4\}}^{1}$ is not fixed under the $\mathbb{C}^{*}$ action from the $R$ symmetry.

This bad hybrid has 2 massless D-branes. The original Calabi-Yau threefold had $h^{1,1}=1$ and $h^{2,1}=89$. At this bad hybrid limit we turn on a deformation of complex structure to smooth the theory and end up with the Gepner model with $h^{1,1}=0$ and $h^{2,1}=90$. It is clear, therefore, that this model is "stuck" at small radius when this 90th deformation is turned on, and this is why there is no natural geometric interpretation.

Having said that, there is a weaker geometrical interpretation in the following sense. The D-brane category associated with the Landau-Ginzburg model (3.5) is the graded category of matrix factorizations of this superpotential [25]. If we call this category $\mathscr{A}$, by a result of Orlov [26] we have a semiorthogonal decomposition

$$
\mathbf{D}^{b}\left(X_{\text {fake }}\right)=\langle\mathscr{A}, \mathscr{O}, \mathscr{O}(1), \mathscr{O}(2), \mathscr{O}(3)\rangle
$$

where $X_{\text {fake }}$ is a quartic hypersurface in $\mathbb{P}_{\{2,1,1,1,1,1,1\}}^{6}$. 
The 5 -fold $X_{\text {fake }}$ has a Hodge diamond

$$
\begin{aligned}
& 1 \\
& \begin{array}{ll}
0 & 0
\end{array} \\
& \begin{array}{lll}
0 & 1 & 0
\end{array} \\
& \begin{array}{llll}
0 & 0 & 0 & 0
\end{array} \\
& \begin{array}{lllll}
0 & 0 & 1 & 0 & 0
\end{array} \\
& \begin{array}{llllll}
0 & 1 & 90 & 90 & 1 & 0
\end{array} \\
& \begin{array}{lllll}
0 & 0 & 1 & 0 & 0
\end{array} \\
& \begin{array}{llll}
0 & 0 & 0 & 0
\end{array} \\
& \begin{array}{llll}
0 & & 1 & \\
& 0 & & 0
\end{array} \\
& 1
\end{aligned}
$$

The middle row is that of a Calabi-Yau threefold with $h^{2,1}=90$ and indeed we expect this to have the same complex structure moduli space as the Landau-Ginzburg theory. However, we wish to emphasize that the extremal transition shows that the more natural physical interpretation is a non-geometric phase stuck at small radius and not $X_{\text {fake }}$.

It is perhaps worth emphasizing a construction which doesn't work. One might be tempted to say the Landau-Ginzburg orbifold associated with the Gepner $\mathbf{2}^{6}$ theory has 6 fields and a superpotential

$$
W=x_{1}^{4}+x_{2}^{4}+x_{3}^{4}+x_{4}^{4}+x_{5}^{4}+x_{6}^{4} .
$$

Correspondingly $X_{\text {fake }}$ would be a quartic 4 -fold in $\mathbb{P}^{5}$. Again we have a category $\mathscr{A}$ of matrix factorizations of (3.8). The problem is that $\mathscr{A}$ is not a Calabi-Yau category. The Serre functor $S_{\mathscr{A}}$ obeys $S_{\mathscr{A}}^{2}=[6]$ but not $S_{\mathscr{A}}=[3] .{ }^{6}$ In other words, the open string spectrum does not exhibit spectral flow. Similarly, the Hodge diamond of the quartic 4fold is not at all what we would want. Adding the $x_{7}^{2}$ term into the superpotential fixes all this, and is what the extremal transition tells us to do.

\subsection{2 $\mathbb{P}_{\{2,1,1,1,1,1\}}^{5} \mid 43$}

Now reconsider the intersection of a cubic and quadric in weighted $\mathbb{P}_{\{2,1,1,1,1,1\}}^{5}$ as in section 3.1. The pointset $\mathscr{A}$ given by the rows of (3.1) have two triangulations. The first is the Calabi-Yau phase as expected. The other is another bad hybrid Landau-Ginzburg fibration over $\mathbb{P}_{\{3,4\}}^{1}$. Just like previous example this has a singular limit at a finite distance in moduli space. We get an extremal transition by removing the point $(1,0,0,0,0,0,0) \in$ $\mathscr{A}$. A generically smooth model is restored by adding the point $(-3,4,3,3,3,-4,-4)$ to $\mathscr{B}$.

The resulting theory has only a Landau-Ginzburg phase with superpotential given by

$$
W=x_{1}^{4}+x_{2}^{4}+x_{3}^{4}+x_{4}^{4}+x_{5}^{4}+b x_{6}^{4}+x_{7}^{2}+\ldots,
$$

\footnotetext{
${ }^{6}$ To see the former use section 4 of [27]. To see the latter use the action of $S_{\mathscr{A}}$ on K-theory as in section 5.1 .6 of $[20]$.
} 
where the extremal transitions "joins on" at $b=0$. That is, the monomial $x_{6}^{4}$ is the extra point we add to $\mathscr{B}$. But this is exactly the same family as the previous example.

So the family of Landau-Ginzburg theories of the form (3.5) (or (3.9)) contains the Gepner model $\mathbf{2}^{6}$ as well as two limit points where the theory degenerates. These have extremal transitions to the two Calabi-Yau threefolds we have considered so far. In particular, we have a path between the non-reflexive example of section 3.1 and the reflexive example of this section.

\subsection{3 $\quad \mathbb{P}^{6} \mid \begin{array}{lll}3 & 2 & 2\end{array}$}

Since we have found an extremal transition to the Gepner model $\mathbf{2}^{6}$, it is worth asking the question if we can find a transition to the well-known, non-geometric Gepner model of $\mathbf{1}^{9}$ which was the first Gepner model constructed [28].

Indeed we can, and it turns out to be an example known to have a singular limit as studied in [16]. The example consists of the complete intersection of two quadrics and a cubic in $\mathbb{P}^{6}$. So the superpotential is

$$
W=x_{8} f_{2}\left(x_{1}, \ldots, x_{7}\right)+x_{9} g_{2}\left(x_{1}, \ldots, x_{7}\right)+x_{10} h_{3}\left(x_{1}, \ldots, x_{6}\right),
$$

where the subscripts on the functions denote their homogeneous degree.

For this example the pointset $\mathscr{A}$ has two triangulations again, with one giving the interpretation as a Calabi-Yau manifold with $h^{1,1}=1$ and $h^{2,1}=73$. The other limit is a bad hybrid with base $\mathbb{P}_{\{3,2,2\}}^{2}$. As discussed in [16], this limit has an $\mathrm{SU}(2)$ gauge symmetry that is inextricably linked to gravity and, as such, has no field theory limit. Interestingly for us, there are also 7 massless fundamentals of $\mathrm{SU}(2)$ in this limit [29]. It follows that there is a Higgs branch of the moduli space giving us an extremal transition.

Repeating the analysis of the previous sections to put this transition into toric language, we lose point from $\mathscr{A}$ corresponding to the variable $x_{10}$ and we gain two points in $\mathscr{B}$ corresponding to the monomials $x_{8}^{3}$ and $x_{9}^{3}$. We have a superpotential which is cubic in 9 variables, and a $\mathbb{Z}_{3}$-orbifold action from $G$. This is the $\mathbf{1}^{9}$ model as promised.

\section{Ignoring interior points}

As we saw in the previous section, ignoring points at the vertices of Conv $\mathscr{A}$ can result in singularities and thus extremal transitions. In this section we consider ignoring points in $\mathscr{A}$ which are not at the vertices. Thus we do not change Conv $\mathscr{A}$ and thus the mode remains generically nonsingular. This has useful applications in studying mirror pairs. In particular, the phase picture becomes very daunting if there are many points in $\mathscr{A}$. By ignoring most of them we still get mirror pairs according to section 2.7 but the phase picture can be more manageable.

An example of this idea which has been done many times implicitly before is to drop points in codimension-one faces for Batyrev-type mirror pairs. After all, it is known that such points do not contribute to the Hodge numbers [4]. We give two other examples of reducing other types of mirror symmetry to that considered in this paper. 


\subsection{Berglund-Hübsch mirror symmetry}

Mirror symmetry exchanges the matrices $A$ and $B$ and therefore takes the transpose of the matrix of exponents in the superpotential $P=A^{t} B$. In the special case that the model in question is a Landau-Ginzburg theory, this should reduce to Berglund-Hübsch [11, 30] mirror symmetry. We describe exactly how this works in a particular example. All other cases are very similar.

Our observations here are certainly not the first concerning the relationship of the Berglund-Hübsch construction to mirror symmetry via toric geometry. See, for example, [31, 32]. The picture we give here has substantial overlap with [33] who first realized that Berglund-Hübsch mirror symmetry arises from a correct understanding of certain phases.

Suppose we have a smooth quintic hypersurface $X$ in $\mathbb{P}^{4}$ defined by

$$
x_{1}^{4} x_{2}+x_{2}^{4} x_{3}+x_{3}^{4} x_{4}+x_{4}^{4} x_{5}+x_{5}^{5} .
$$

(This example first appeared in [34].) We completely understand mirror symmetry for quintics and so we should certainly be able to construct the mirror. In particular, we have the explicit mirror map (2.40) so we should know exactly which Kähler data on the mirror corresponds to the choice of complex structure (4.1). It follows that we should know which phase the mirror is in.

\subsubsection{A reflexive model}

The standard way to proceed is to use the well-known standard reflexive pair of polytopes for the quintic and its mirror. The data for the mirror is

$$
A^{t}=\left(\begin{array}{ccccc}
1 & 4 & -1 & -1 & -1 \\
1 & -1 & 4 & -1 & -1 \\
1 & -1 & -1 & 4 & -1 \\
1 & -1 & -1 & -1 & 4 \\
1 & -1 & -1 & -1 & -1 \\
\vdots &
\end{array}\right), B^{t}=\left(\begin{array}{ccccc}
1 & 0 & 0 & 0 & 0 \\
1 & 1 & 0 & 0 & 0 \\
1 & 0 & 1 & 0 & 0 \\
1 & 0 & 0 & 1 & 0 \\
1 & 0 & 0 & 0 & 1 \\
1 & -1 & -1 & -1 & -1
\end{array}\right)
$$

The pointset $\mathscr{A}$ has 126 points and so we only show those at the vertices of convex hull above. With this many points, an analysis of the phase picture is hopeless. What we do instead is choose $\mathscr{A}$ to be the 5 points which are the vertices of Conv $\mathscr{A}$ together with 4 more points given by the first 4 monomials in (4.1). We are therefore considering mirror of the family of quintics whose defining equation has 9 monomials - the union of the Fermat ones and those in (4.1). The resulting pointset has a more manageable 42 triangulations. One obvious triangulation consists of the single simplex given by the convex hull. This is mirror to the familiar Landau-Ginzburg theory $\mathbb{C}^{5} /\left(\mathbb{Z}_{5}^{\oplus 4}\right)$.

Four of the triangulations contain a simplex with vertices corresponding to the monomials in (4.1). Each of these is consistent with varying the complex structure so that we return to the defining equation (4.1). We show such a triangulation schematically (in one fewer dimensions) on the left in figure 4 . Now consider the toric variety $Z_{\Sigma}$ associated 

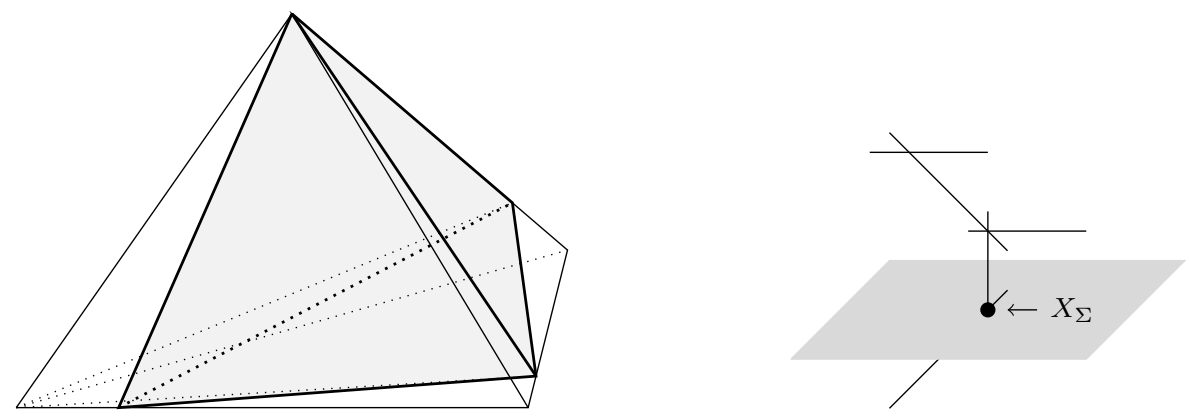

Figure 4. A Hidden Landau-Ginzburg Theory.

to one such triangulation. It is certainly not of the form $\mathbb{C}^{5} / H$, for some finite group $H$, as one would normally expect for a Landau-Ginzburg phase. Instead the set of compact $T_{N}$-orbits comprise a surface and set of $\mathbb{P}^{1}$ 's as shown on the right in figure 4 . However, when one computes the critical points of $W$ to compute $X_{\Sigma}$, one finds that this is a single point. So the theory really is a Landau-Ginzburg orbifold theory. Usually one associates a Landau-Ginzburg phase with a triangulation consisting of a single simplex, so this might be regarded as a "hidden" Landau-Ginzburg phase.

Locally, at the point $X_{\Sigma}$, the geometry of $V_{\Sigma}$ is $\mathbb{C}^{5} / \mathbb{Z}_{256}$ with group generator acting with weights $\frac{1}{256}(1,251,20,176,64)$. We have therefore shown that the mirror of the quintic hypersurface with defining equation (4.1) is a Landau-Ginzburg orbifold of the form $\mathbb{C}^{5} / \mathbb{Z}_{256}$. The superpotential is the transposed form of (4.1):

$$
x_{1}^{5} x_{2}+x_{2}^{4} x_{3}+x_{3}^{4} x_{4}+x_{4}^{4} x_{5}+x_{5}^{4} .
$$

We can produce a geometric mirror by deforming the complex structure of the quintic by adding the monomial $x_{1} x_{2} x_{3} x_{4} x_{5}$ to (4.1) with a large coefficient. In the mirror, on the Kähler side, this amounts to including the point $(1,0,0,0,0)$ in the triangulation. This blows up the $\mathbb{C}^{5} / \mathbb{Z}_{256}$ singularity and $X_{\Sigma}$ becomes a degree 256 hypersurface in $\mathbb{P}_{\{41,48,51,52,64\}}^{4}$.

We claim that all Berglund-Hübsch examples arise this way. In particular any defining equation with only 5 terms that leads to a smooth Calabi-Yau will be mirror to a LandauGinzburg orbifold theory. The five monomials become vertices of a 4 -simplex in $\mathscr{A}$ and the smoothness condition guarantees that the critical pointset of the superpotential is an isolated point.

\subsubsection{A non-reflexive version}

Now let's do the same analysis in a more direct way. Simply set $\mathscr{A}$ to only 5 points given by the monomials in (4.1). That is

$$
A^{t}=\left(\begin{array}{ccccc}
1 & -1 & -1 & -1 & -1 \\
1 & 3 & 0 & -1 & -1 \\
1 & -1 & 3 & 0 & -1 \\
1 & -1 & -1 & 3 & 0 \\
1 & -1 & -1 & -1 & 3
\end{array}\right)
$$


The dual of the cone over Conv $\mathscr{A}$ is then generated by the rows of the following matrix:

$$
\left(\begin{array}{ccccc}
1 & -51 / 41 & -52 / 41 & -48 / 41 & -64 / 41 \\
1 & 1 & 0 & 0 & 0 \\
1 & -1 / 3 & 4 / 3 & 0 & 0 \\
1 & 1 / 13 & -4 / 13 & 16 / 13 & 0 \\
1 & -1 / 51 & 4 / 51 & -16 / 51 & 64 / 51
\end{array}\right)
$$

Now intersect this cone with the lattice $M$ and the hyperplane $H_{\nu}$, where $\nu=(1,0,0,0,0)$. The result is the pointset $\mathscr{B}$ given by rows of the matrix

$$
B^{t}=\left(\begin{array}{ccccc}
1 & 0 & 0 & 0 & 0 \\
1 & 1 & 0 & 0 & 0 \\
1 & 0 & 1 & 0 & 0 \\
1 & 0 & 0 & 1 & 0 \\
1 & 0 & 0 & 0 & 1 \\
1 & -1 & -1 & -1 & -1
\end{array}\right)
$$

which is, of course, the data for the quintic. Thus we recover the quintic as above. We only have one phase, a Landau-Ginzburg phase on $\mathbb{C}^{5} / \mathbb{Z}_{256}$ as above. Note that we do not have any of the irrelevant detritus sticking out of the Landau-Ginzburg theory as we had in figure 4.

This is a perfectly good gauged linear $\sigma$-model. It is $\mathscr{B}$-complete but not $\mathscr{A}$-complete. So the conditions in conjecture 10 do not give necessary conditions for a generically nonsingular family of models.

\subsection{Vafa-Witten mirror symmetry}

Perhaps a little gratuitously, let us give another example of dropping internal points to simplify mirror symmetry. In [12] a mirror pair of orbifolds were constructed using discrete torsion. Let $E$ be an elliptic curve and let $X^{\sharp}$ be the orbifold $(E \times E \times E) / H$, where $H=\mathbb{Z}_{2} \times \mathbb{Z}_{2}$ generated by $(-1,-1,1)$ and $(1,-1,-1) . X^{\sharp}$ may be blown-up to a smooth Calabi-Yau threefold $X$ with $h^{1,1}=51$ and $h^{2,1}=3$.

Since $H^{2}(H, \mathrm{U}(1))=\mathbb{Z}_{2}$, we may consider the orbifold with discrete torsion switched on. It [12] it was argued that the resulting theory is mirror to the orbifold without discrete torsion. It was also argued that a generic member of the resulting family, $Y$, mirror to $X$, has 64 "stuck" nodes. This was also analyzed in [35] in terms of the Brauer group. This Brauer group interpretation was extended to other models in [36, 37] in a way relevant for us here.

Let the elliptic $E$ be realized as a quartic $x_{1}^{2}+x_{2}^{4}+x_{3}^{4}$ in $\mathbb{P}_{\{2,1,1\}}^{2}$. Then " -1 " acts on $E$ as $\left[x_{1}, x_{2}, x_{3}\right] \mapsto\left[-x_{1}, x_{2}, x_{3}\right]$. In our desired gauged linear $\sigma$-model language we will write this as the set of points

$$
A^{t}=\left(\begin{array}{ccc}
1 & 0 & 0 \\
1 & 1 & 0 \\
1 & 0 & 1 \\
1 & -2 & -1
\end{array}\right) \text {. }
$$




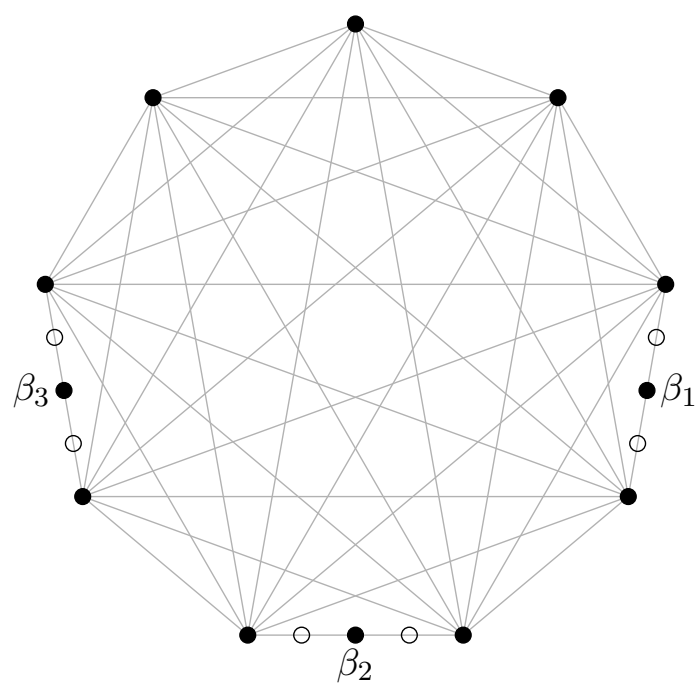

Figure 5. The Pointset $\mathscr{B}$ for the orbifold $T^{6} / \mathbb{Z}_{2} \times \mathbb{Z}_{2}$.

The " -1 " is part of the torus $T_{N}$ and thus can also be written as a vector in $N \otimes \mathbb{Q}$. This vector is $\left(\frac{1}{2}, \frac{1}{2}, 0\right)$.

To build $E \times E \times E$ we simply take the direct sum of three copies of (4.7) so that $N$ is now 9 -dimensional. The action of $H$ is then generated by

$$
\begin{aligned}
& \left(\frac{1}{2}, \frac{1}{2}, 0, \frac{1}{2}, \frac{1}{2}, 0,0,0,0\right) \\
& \left(0,0,0, \frac{1}{2}, \frac{1}{2}, 0, \frac{1}{2}, \frac{1}{2}, 0\right)
\end{aligned}
$$

To perform the orbifold in toric language, we fix the pointset $\mathscr{A}$ but modify the lattice $N$ by refining it minimally to include the two vectors (4.8). One then transforms the matrix $A$ according to a change of basis to one that generates the new $N$.

This results in a reflexive pair of points $\mathscr{A}$ and $\mathscr{B}$ so we should know exactly what the mirror of $X$ is. The convex hull of both $\mathscr{A}$ and $\mathscr{B}$ are simplices with 9 vertices. That means they both have a phase that is purely a Landau-Ginzburg orbifold. Indeed, they are both orbifolds of the Gepner model $2^{6}$ and so this mirror symmetry is actually of the Greene-Plesser type [38].

The pointset $\mathscr{B}$ in this 8 -simplex is shown in figure 5 . There are 18 points with 9 at the vertices of the simplex. From now on we wish to describe the mirror of $X$ and thus we exchange the roles of $\mathscr{A}$ and $\mathscr{B}$. Points in figure 5 now correspond to homogeneous coordinates.

We will choose to ignore the 6 points shown as unfilled circles. The remaining 12 points have 8 triangulations. These 8 triangulations are easy to understand; one can either include or omit from the triangulation each of the 3 points shown as $\beta_{1}, \beta_{2}, \beta_{3}$ in the figure. 
The superpotential in these 12 variables can be written

$$
W=\left(\begin{array}{lll}
x_{1} & x_{2} & x_{3}
\end{array}\right) M\left(\begin{array}{l}
x_{1} \\
x_{2} \\
x_{3}
\end{array}\right)+x_{10}^{2}+x_{11}^{2}+x_{12}^{2}
$$

where

$$
M=\left(\begin{array}{lll}
f_{400} & f_{220} & f_{202} \\
f_{220} & f_{040} & f_{022} \\
f_{202} & f_{022} & f_{004}
\end{array}\right),
$$

and $f_{i j k}$ is a homogeneous function of degree $(i, j, k)$ in the variables $\left(x_{4}, x_{5}\right),\left(x_{6}, x_{7}\right)$, $\left(x_{8}, x_{9}\right)$ respectively. The notation is chosen to coincide with that of [35].

In the triangulation where we include all three points $\beta_{1}, \beta_{2}, \beta_{3}, V_{\Sigma}$ is a vector bundle over $\mathbb{P}^{1}\left[x_{4}, x_{5}\right] \times \mathbb{P}^{1}\left[x_{6}, x_{7}\right] \times \mathbb{P}^{1}\left[x_{8}, x_{9}\right]$. We therefore have a hybrid model with base $\mathbb{P}^{1} \times \mathbb{P}^{1} \times \mathbb{P}^{1}$ and fibre given by a quadratic Landau-Ginzburg theory. This situation is essentially identical to the model of 4 quadrics in $\mathbb{P}^{7}$ which has been extensively analyzed in $[8,36,37,39]$. Accordingly, we interpret this hybrid model as a double cover of $\mathbb{P}^{1} \times \mathbb{P}^{1} \times \mathbb{P}^{1}$ branched over the surface given by $\operatorname{det} M=0$. This surface has 64 nodes as does the double cover. A Brauer class is "switched on" in the same way as it occurs in [37]. This precisely agrees with Vafa and Witten's mirror [12].

It is worth mentioning the other phases. Consider the phase where we include $\beta_{1}$ but not $\beta_{2}$ or $\beta_{3}$ in the triangulation. This is a hybrid model with base $\mathbb{P}^{1}\left[x_{4}, x_{5}\right]$. To see the fibre, fix the values of $x_{4}$ and $x_{5}$ which gives a Landau-Ginzburg superpotential quartic in $x_{6}, x_{7}, x_{8}, x_{9}$ and quadratic in $x_{1}, x_{2}, x_{3}, x_{10}, x_{11}, x_{12}$. The usual arguments go that we can ignore the quadratic terms in a superpotential ${ }^{7}$ leaving us with a quartic in four variables. This, of course is equivalent to a quartic K3 surface and thus we appear to have a K3-fibration.

The problem is that there are 4 points on the base $\mathbb{P}^{1}$ where the rank of $M$ drops and the variables we were ignoring have a vanishing coefficient in their quadratic term. Thus, at the bad fibres, it is these ignored variables that do something interesting, and not the K3 part. It follows that we do not have a family of K3's near the bad fibres, and so we do not have a K3 fibration. Similarly we have a broken elliptic fibration over $\mathbb{P}^{1} \times \mathbb{P}^{1}$ in some of the other phases.

\section{Discussion}

Clearly it would be nice to find a proof of, or counterexample to, our conjectured sufficient condition for a generically nonsingular model. An obvious way to find a counterexample is to search examples via a random walk through extremal transitions. Indeed, the formulation of the conjecture is well-suited to such a search. It would also be very nice to find a necessary condition rather than a sufficient condition. We should note that when Conv $\mathscr{A}$

\footnotetext{
${ }^{7}$ To get the open string spectrum correct we use Knörrer periodicity, which says we can ignore an even number of variables appearing quadratically [26].
} 
is a simplex, the work of [40] effectively solves this problem. The more general situation is, presumably, even more complicated.

If the conjecture is true, a search may generate many more examples of nonreflexive mirror pairs. The famous list [41] of reflexive pairs for the hypersurface case (i.e., $\langle\mu, \nu\rangle=1$ ) runs to half a billion. Progress beyond this special case has been limited, but has generally involved notions of "nef partitions" following [5]. If one simply wants to enumerate possible string compactifications or Calabi-Yau categories then this notion is irrelevant. A full enumeration might be simply too large to practically achieve, but it would be interesting to see if certain properties are common. For example, how likely is it that any geometric phase exists for a given model? A large-radius limit may, or may not, arise as a geometric phase limit as discussed in 2.4, so a separate question would be how likely is it that a large-radius limit exists for a given model.

The simple way in which (2.40) implements mirror symmetry is in a sense surprising. There is, for example, no a priori reason from the point of view of superconformal field theory why mirror symmetry should preserve the toric subspace. This suggestive structure led to the idea that mirror symmetry can be simply implemented as a T-duality transformation directly in the gauged linear $\sigma$-model $[6,7]$. This duality would imply that if one can write a condition on the combinatorial data that is both necessary and sufficient for the gauged linear $\sigma$-model to be generically nonsingular it too should be mirror symmetric. Finding this is not likely to be straightforward, since in particular it would involve refining the GKZ determinant to a necessary and sufficient condition, but examples like 4.1.2 may be helpful as nontrivial test cases.

Our construction of the GKZ determinant (2.26) from the gauged linear $\sigma$-model is not quite complete. We have not derived the powers $u(\Gamma)$. A more complete analysis of the model based on $\mathscr{A}_{h}$ in section 2.6.2 should surely explain these numbers. This should be investigated further.

\section{Acknowledgments}

We thank Nick Addington, David Favero, Ilarion Melnikov and David Morrison for helpful discussions. PSA is supported by NSF grants DMS-1207708. MRP is supported by NSF grant PHY-1217109. Any opinions, findings, and conclusions or recommendations expressed in this material are those of the authors and do not necessarily reflect the views of the National Science Foundation.

Open Access. This article is distributed under the terms of the Creative Commons Attribution License (CC-BY 4.0), which permits any use, distribution and reproduction in any medium, provided the original author(s) and source are credited.

\section{References}

[1] E. Witten, Phases of $N=2$ theories in two-dimensions, Nucl. Phys. B 403 (1993) 159 [hep-th/9301042] [INSPIRE]. 
[2] P.S. Aspinwall, B.R. Greene and D.R. Morrison, Calabi-Yau moduli space, mirror manifolds and space-time topology change in string theory, Nucl. Phys. B 416 (1994) 414 [hep-th/9309097] [INSPIRE].

[3] D.R. Morrison and M.R. Plesser, Summing the instantons: quantum cohomology and mirror symmetry in toric varieties, Nucl. Phys. B 440 (1995) 279 [hep-th/9412236] [INSPIRE].

[4] V.V. Batyrev, Dual polyhedra and mirror symmetry for Calabi-Yau hypersurfaces in toric varieties, J. Alg. Geom. 3 (1994) 493 [alg-geom/9310003] [INSPIRE].

[5] L. Borisov, Towards the mirror symmetry for Calabi-Yau complete intersections in Gorenstein toric Fano varieties, alg-geom/9310001.

[6] D.R. Morrison and M.R. Plesser, Towards mirror symmetry as duality for two-dimensional Abelian gauge theories, Nucl. Phys. Proc. Suppl. 46 (1996) 177 [hep-th/9508107] [INSPIRE].

[7] K. Hori and C. Vafa, Mirror symmetry, hep-th/0002222 [INSPIRE].

[8] P.S. Aspinwall and B.R. Greene, On the geometric interpretation of $N=2$ superconformal theories, Nucl. Phys. B 437 (1995) 205 [hep-th/9409110] [INSPIRE].

[9] A.C. Avram, M. Kreuzer, M. Mandelberg and H. Skarke, The web of Calabi-Yau hypersurfaces in toric varieties, Nucl. Phys. B 505 (1997) 625 [hep-th/9703003] [INSPIRE].

[10] I.M. Gelfand, M.M. Kapranov and A.V. Zelevinski, Discriminants, resultants and multidimensional determinants, Birkhäuser, Boston MA U.S.A. (1994).

[11] P. Berglund and T. Hubsch, A generalized construction of mirror manifolds, Nucl. Phys. B 393 (1993) 377 [hep-th/9201014] [INSPIRE].

[12] C. Vafa and E. Witten, On orbifolds with discrete torsion, J. Geom. Phys. 15 (1995) 189 [hep-th/9409188] [INSPIRE].

[13] D.A. Cox, J.B. Little and H.K. Schenck, Toric varities, Graduate Studies in Mathematics 124, Amer. Math. Soc., Providence RI U.S.A. (2010).

[14] P.S. Aspinwall, I.V. Melnikov and M.R. Plesser, (0,2) elephants, JHEP 01 (2012) 060 [arXiv: 1008.2156] [INSPIRE].

[15] L.A. Borisov, L. Chen and G.G. Smith, The orbifold Chow ring of toric Deligne-Mumford stacks, J. Amer. Math. Soc. 18 (2005) 193 [math/0309229].

[16] P.S. Aspinwall and M.R. Plesser, Decompactifications and massless D-branes in hybrid models, JHEP 07 (2010) 078 [arXiv: 0909. 0252] [INSPIRE].

[17] E. Witten, Mirror manifolds and topological field theory, in Essays on mirror manifolds, S.-T. Yau ed., International Press, (1992) [hep-th/9112056] [INSPIRE].

[18] V. Batyrev and B. Nill, Combinatorial aspects of mirror symmetry, in Integer points in polyhedra - geometry, number theory, representation theory, algebra, optimization, statistics, Contemp. Math. 452, Amer. Math. Soc., Providence RI U.S.A. (2008), pg. 35 [math/0703456] [INSPIRE].

[19] B.R. Greene, D.R. Morrison and C. Vafa, A geometric realization of confinement, Nucl. Phys. B 481 (1996) 513 [hep-th/9608039] [INSPIRE].

[20] N. Addington and P.S. Aspinwall, Categories of massless D-branes and del Pezzo surfaces, JHEP 07 (2013) 176 [arXiv: 1305. 5767] [INSPIRE]. 
[21] P. Candelas, E. Derrick and L. Parkes, Generalized Calabi-Yau manifolds and the mirror of a rigid manifold, Nucl. Phys. B 407 (1993) 115 [hep-th/9304045] [INSPIRE].

[22] P.S. Aspinwall, B.R. Greene and D.R. Morrison, The monomial divisor mirror map, Internat. Math. Res. Notices 1993319 [alg-geom/9309007] [INSPIRE].

[23] A. Strominger, Massless black holes and conifolds in string theory, Nucl. Phys. B 451 (1995) 96 [hep-th/9504090] [INSPIRE].

[24] L.J. Billera, P. Filliman and B. Sturmfels, Constructions and complexity of secondary polytopes, Adv. Math. 83 (1990) 155.

[25] K. Hori and J. Walcher, D-branes from matrix factorizations, Comptes Rendus Physique $\mathbf{5}$ (2004) 1061 [hep-th/0409204] [INSPIRE].

[26] D. Orlov, Derived categories of coherent sheaves and triangulated categories of singularities, in Algebra, arithmetic, and geometry: in honor of Yu.I. Manin. Vol. II, Progr. Math. 270, Birkhäuser, Boston MA U.S.A. (2009), pg. 503 [math/0503632].

[27] A.G. Kuznetsov, Derived category of a cubic threefold and the variety $V_{14}$, Tr. Mat. Inst. Steklova 246 (2004) 183 [math/0303037].

[28] D. Gepner, Exactly solvable string compactifications on manifolds of $\mathrm{SU}(N)$ holonomy, Phys. Lett. B 199 (1987) 380 [INSPIRE].

[29] N. Addington and P.S. Aspinwall, to appear.

[30] M. Krawitz, FJRW rings and Landau-Ginzburg mirror symmetry, arXiv:0906.0796.

[31] L.A. Borisov, Berglund-Hübsch mirror symmetry via vertex algebras, Commun. Math. Phys. 320 (2013) 73 [arXiv: 1007.2633] [INSPIRE].

[32] P. Clarke, Duality for toric Landau-Ginzburg models, arXiv:0803.0447 [INSPIRE].

[33] D. Favero and T.L. Kelly, Toric mirror constructions and derived equivalence, arXiv: 1412.1354 [INSPIRE].

[34] B.R. Greene and M.R. Plesser, Mirror manifolds: a brief review and progress report, hep-th/9110014 [INSPIRE].

[35] P.S. Aspinwall, D.R. Morrison and M. Gross, Stable singularities in string theory, Commun. Math. Phys. 178 (1996) 115 [hep-th/9503208] [INSPIRE].

[36] A. Caldararu, J. Distler, S. Hellerman, T. Pantev and E. Sharpe, Non-birational twisted derived equivalences in Abelian GLSMs, Commun. Math. Phys. 294 (2010) 605 [arXiv: 0709.3855] [INSPIRE].

[37] N. Addington, The derived category of the intersection of four quadrics, arXiv:0904.1764.

[38] B.R. Greene and M.R. Plesser, Duality in Calabi-Yau moduli space, Nucl. Phys. B 338 (1990) 15 [INSPIRE].

[39] A. Kuznetsov, Derived categories of quadric fibrations and intersections of quadrics, Adv. Math. 218 (2008) 1340 [math/0510670].

[40] M. Kreuzer and H. Skarke, On the classification of quasihomogeneous functions, Commun. Math. Phys. 150 (1992) 137 [hep-th/9202039] [INSPIRE].

[41] M. Kreuzer and H. Skarke, Complete classification of reflexive polyhedra in four-dimensions, Adv. Theor. Math. Phys. 4 (2002) 1209 [hep-th/0002240] [INSPIRE]. 